\title{
A comparison of the Standard 6-31G and Binning-Curtiss \\ Basis Sets for Third Row Elements
}

\author{
Shahidul M. Islam, Stephanie D. Huelin, Margot Dawe \\ and Raymond A. Poirier*
}

Department of Chemistry, Memorial University, St. John's, Newfoundland, Canada A1B 3X7

Tel.: (709) 737-8609

Fax: (709) 737-3702

E-mail: rpoirier@mun.ca

\section{Supplementary Data:}

All the optimized structures for individual molecules are provided along with the level of theory and basis set. For all the structures, total energies, nuclear repulsion energies and symmetry point groups are also provided. 


\section{STRUCTURES OPTIMIZED USING BINNING-CURTISS (BC6-31G) BASIS SET :}

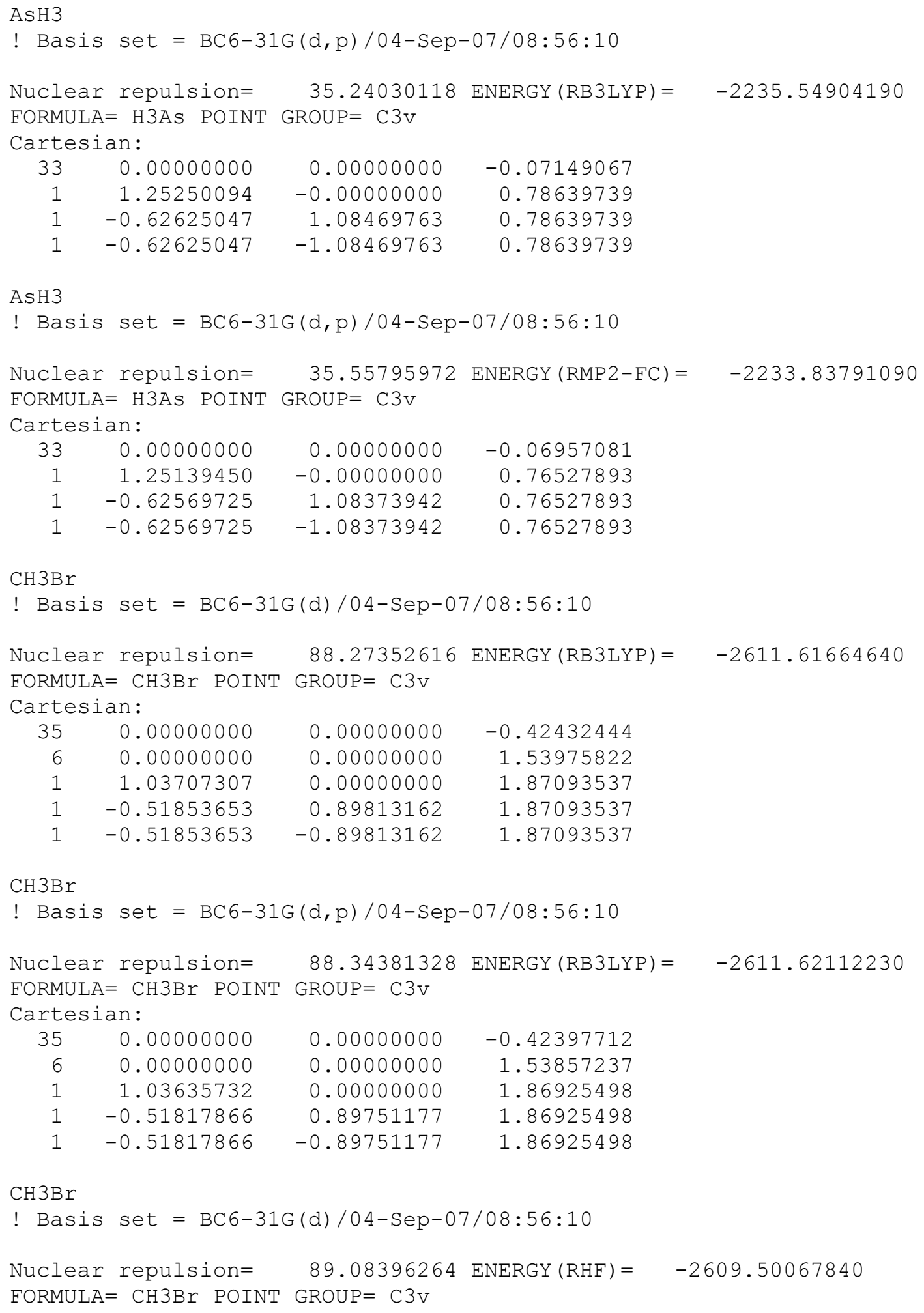




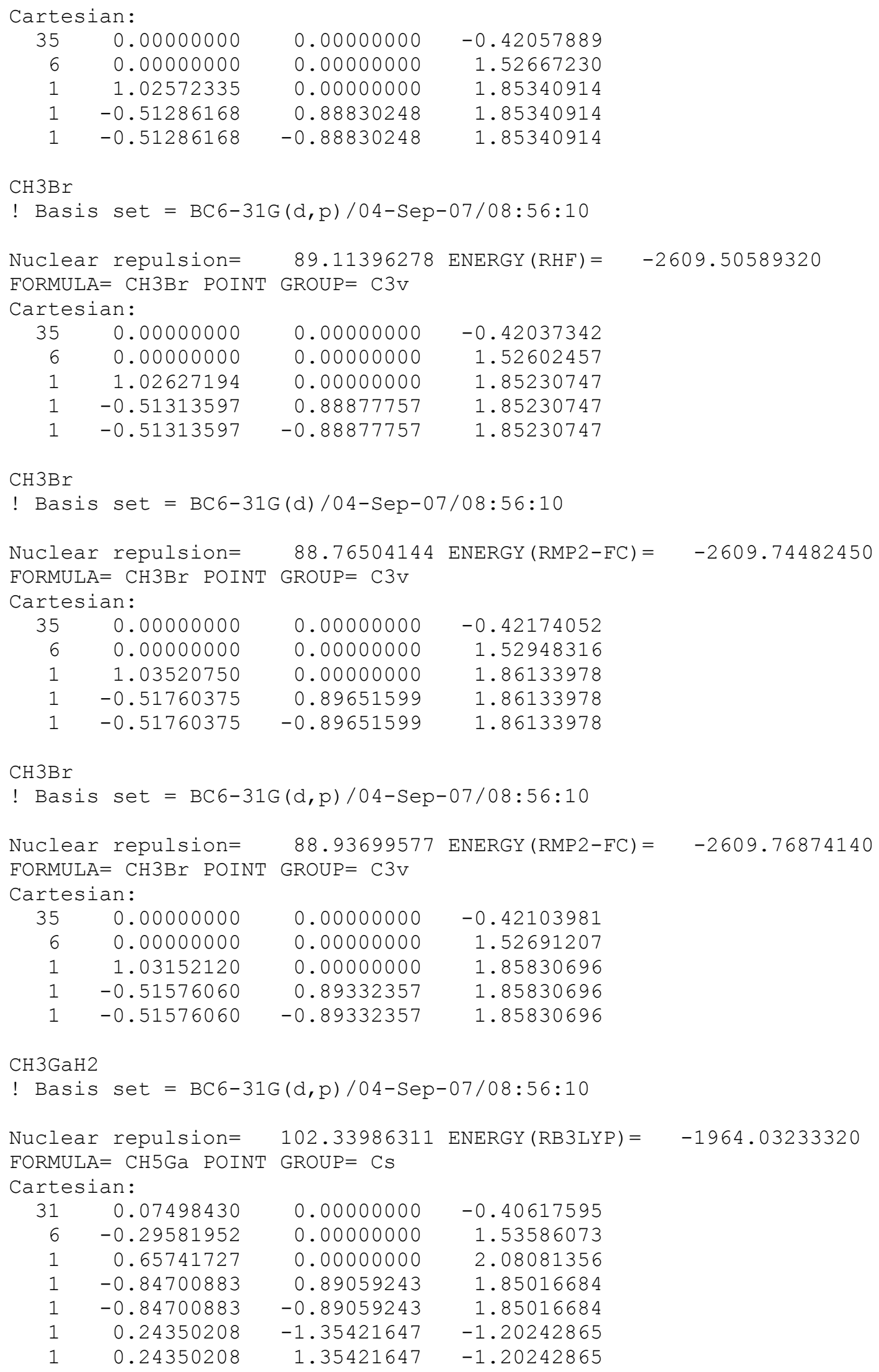




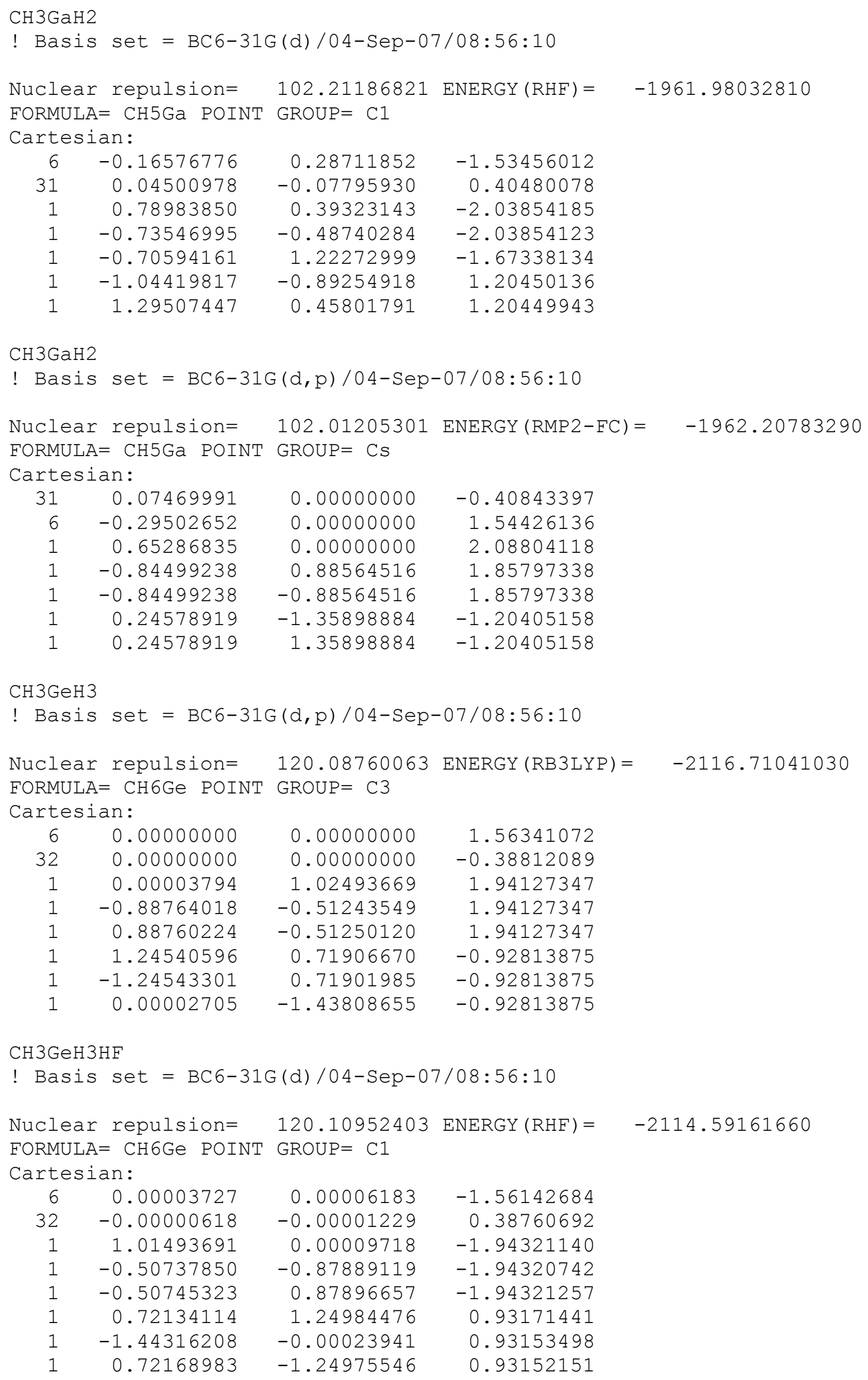

$\begin{array}{rrrr}6 & 0.00003727 & 0.00006183 & -1.56142684 \\ 32 & -0.00000618 & -0.00001229 & 0.38760692 \\ 1 & 1.01493691 & 0.00009718 & -1.94321140 \\ 1 & -0.50737850 & -0.87889119 & -1.94320742 \\ 1 & -0.50745323 & 0.87896657 & -1.94321257 \\ 1 & 0.72134114 & 1.24984476 & 0.93171441 \\ 1 & -1.44316208 & -0.00023941 & 0.93153498 \\ 1 & 0.72168983 & -1.24975546 & 0.93152151\end{array}$




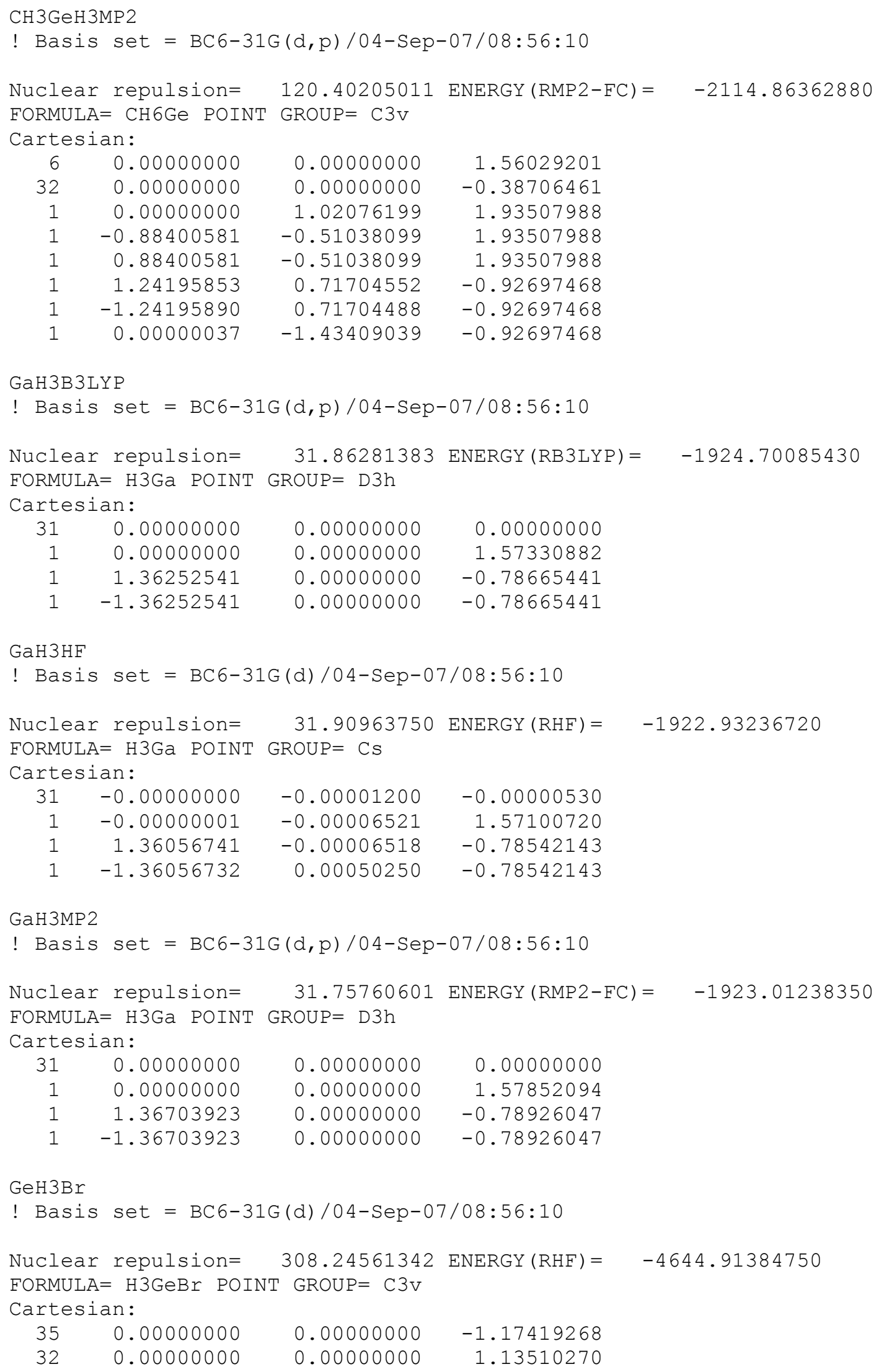




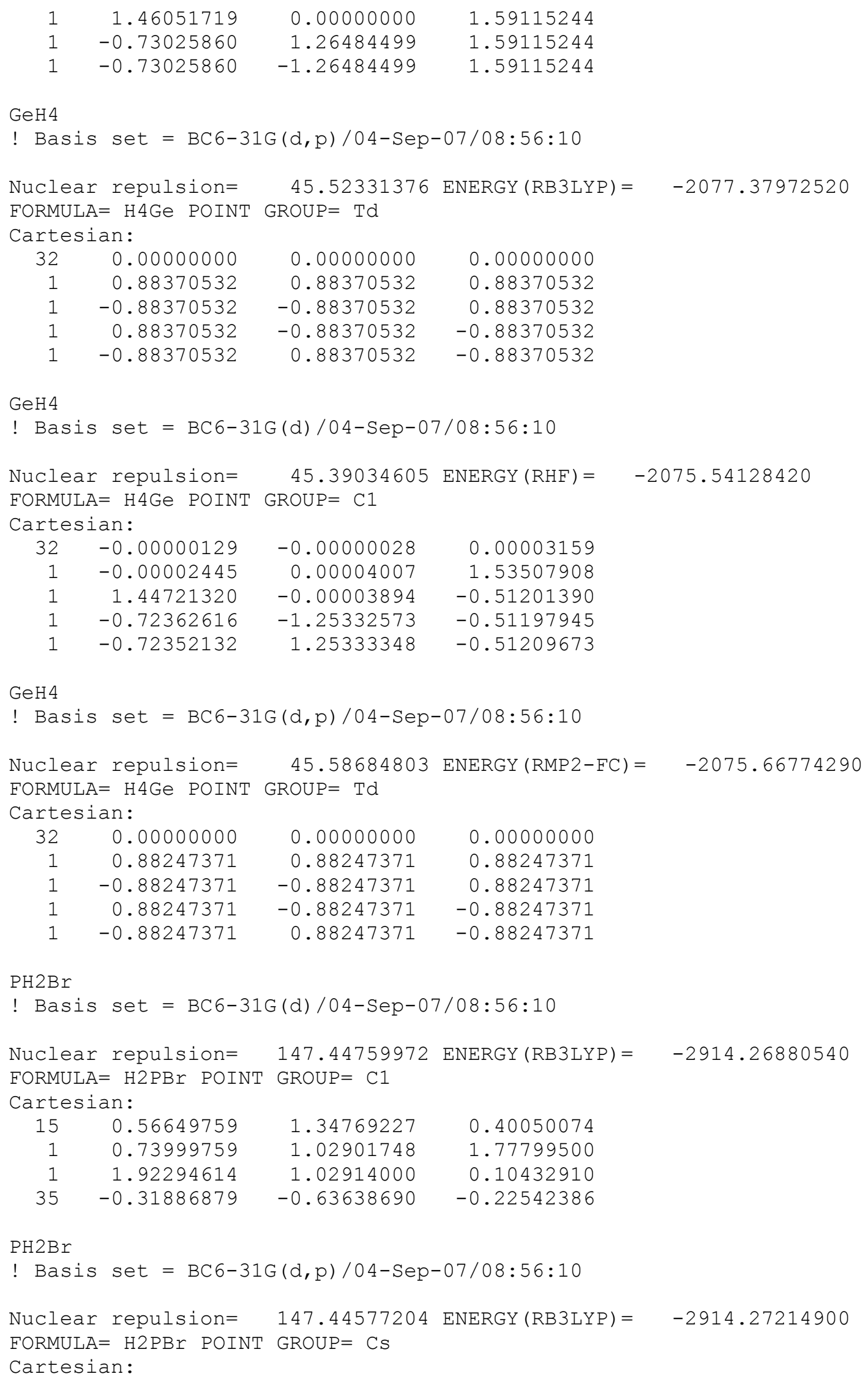




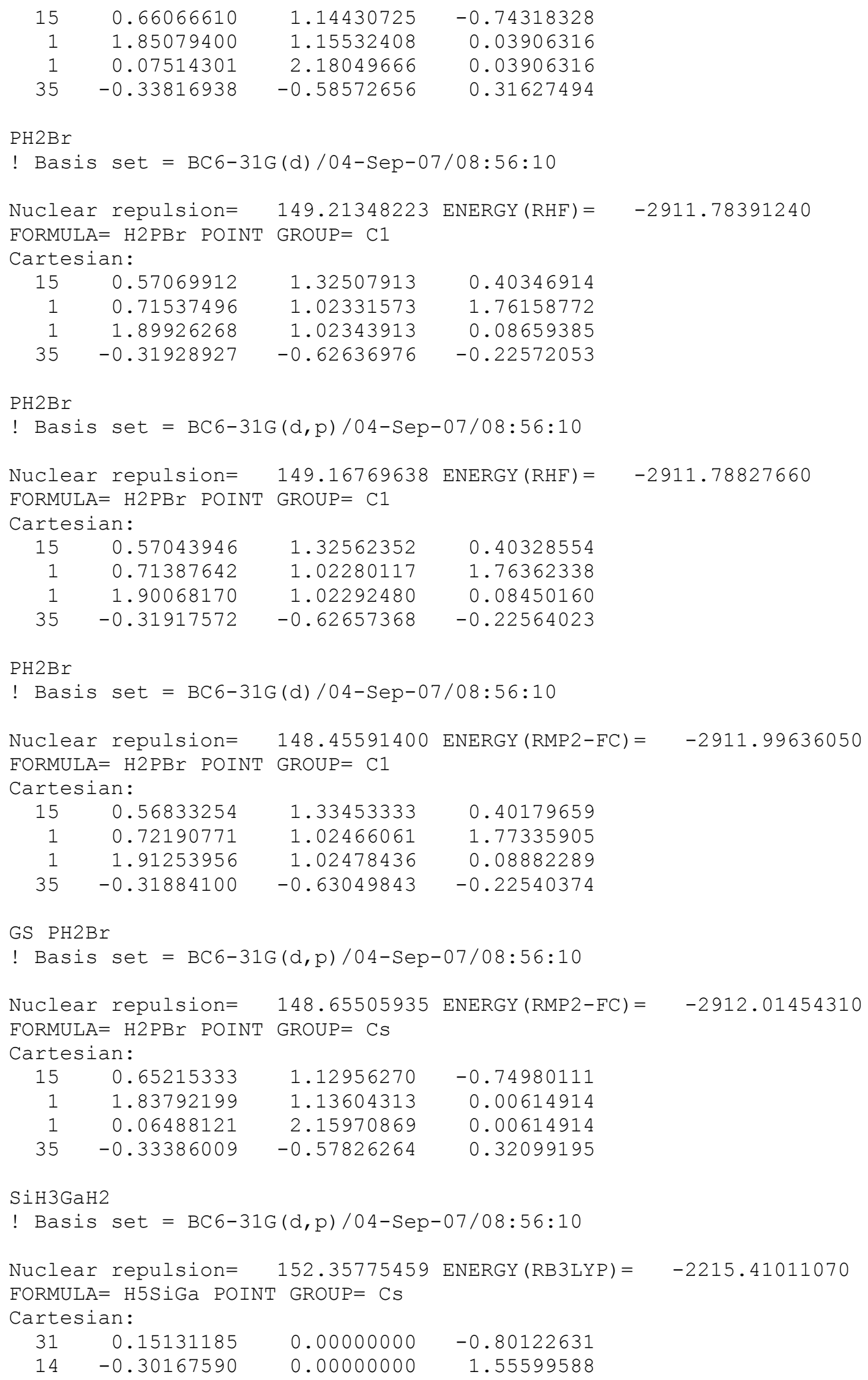




$\begin{array}{rrrr}1 & 0.99767454 & 0.00000000 & 2.29111146 \\ 1 & -1.05878760 & 1.20583080 & 1.99204689 \\ 1 & -1.05878760 & -1.20583080 & 1.99204689 \\ 1 & 0.32634792 & -1.34437230 & -1.61056598 \\ 1 & 0.32634792 & 1.34437230 & -1.61056598\end{array}$

\begin{tabular}{|c|c|c|c|}
\hline \\
\hline \multicolumn{4}{|c|}{ ! Basis set $=B C 6-31 G(d) / 04-$ Sep $-07 / 08: 56: 10$} \\
\hline \multirow{3}{*}{\multicolumn{4}{|c|}{$\begin{array}{l}\text { Nuclear repulsion }=151.31702658 \text { ENERGY }(\mathrm{RHF})= \\
\text { FORMULA }=\text { H5SiGa POINT GROUP }=\mathrm{Cs} \\
\text { Cartesian: }\end{array}$}} \\
\hline & & & \\
\hline & & & \\
\hline & -0.00076238 & -0.00128871 & -1.60300172 \\
\hline & -0.00090187 & -0.00152444 & 0.82441844 \\
\hline & 1.38805364 & 0.00039492 & -2.11837902 \\
\hline & -0.69526559 & -1.17522681 & -2.17500935 \\
\hline & -0.66798815 & 1.21675174 & -2.11837904 \\
\hline 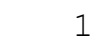 & -0.67744503 & -1.14510416 & 1.67254080 \\
\hline 1 & 0.69127644 & 1.16848384 & 1.62427897 \\
\hline
\end{tabular}

$\mathrm{SiH} 3 \mathrm{GaH} 2$

! Basis set $=B C 6-31 G(d, p) / 04-S e p-07 / 08: 56: 10$

Nuclear repulsion $=\quad 151.59673690$ ENERGY $($ RMP2 - FC $)=\quad-2213.19417180$ FORMULA $=$ H5SiGa POINT GROUP $=\mathrm{CS}$

Cartesian:

$\begin{array}{rrrr}31 & 0.15144622 & 0.00000000 & -0.80732713 \\ 14 & -0.30097935 & 0.00000000 & 1.56987184 \\ 1 & 0.98734320 & 0.00000000 & 2.30155412 \\ 1 & -1.05700556 & 1.19854464 & 1.99104981 \\ 1 & -1.05700556 & -1.19854464 & 1.99104981 \\ 1 & 0.32277302 & -1.34917296 & -1.61735919 \\ 1 & 0.32277302 & 1.34917296 & -1.61735919\end{array}$

SiH3GeH3

! Basis set $=B C 6-31 G(d, p) / 04-S e p-07 / 08: 56: 10$

Nuclear repulsion $=\quad 172.75781154$ ENERGY $($ RB3LYP $)=\quad-2368.08977840$ FORMULA $=$ H6SiGe POINT GROUP $=\mathrm{C} 3$

Cartesian:

$\begin{array}{rrrr}14 & 0.00000000 & 0.00000000 & 1.60322601 \\ 32 & 0.00000000 & 0.00000000 & -0.77624018 \\ 1 & 0.00004446 & 1.39401434 & 2.12017184 \\ 1 & -1.20727406 & -0.69696866 & 2.12017184 \\ 1 & 1.20722960 & -0.69704568 & 2.12017184 \\ 1 & 1.24447433 & 0.71852021 & -1.32199794 \\ 1 & -1.24449392 & 0.71848627 & -1.32199794 \\ 1 & 0.00001959 & -1.43700649 & -1.32199794\end{array}$

$\mathrm{SiH} 3 \mathrm{GeH} 3$

! Basis set $=$ BC6-31G(d)/04-Sep-07/08:56:10

Nuclear repulsion $=\quad 172.23474612$ ENERGY $($ RHF $)=\quad-2365.63489280$ FORMULA $=$ H6SiGe POINT GROUP $=\mathrm{C} 1$

Cartesian:
14
0.00007497
$-0.00002043$
$-1.61074048$
32
$-0.00003388$
0.00000275
0.77954783 


$\begin{array}{rrrr}1 & 1.38568798 & -0.00007755 & -2.12145161 \\ 1 & -0.69279360 & -1.19990762 & -2.12157251 \\ 1 & -0.69263151 & 1.20001986 & -2.12143842 \\ 1 & 0.72242594 & 1.25167179 & 1.32304758 \\ 1 & -1.44526773 & -0.00005416 & 1.32297888 \\ 1 & 0.72261354 & -1.25145417 & 1.32327230\end{array}$

SiH3GeH3

! Basis set $=B C 6-31 G(d, p) / 04-$ Sep-07/08:56:10

Nuclear repulsion $=\quad 172.86875448$ ENERGY (RMP2-FC) $=\quad-2365.85169940$ FORMULA $=$ H6SiGe POINT GROUP $=\mathrm{C} 3$

Cartesian:

$\begin{array}{rrrr}14 & 0.00000000 & 0.00000000 & 1.60579890 \\ 32 & 0.00000000 & 0.00000000 & -0.77695327 \\ 1 & 0.00004129 & 1.38616527 & 2.11215379 \\ 1 & -1.20047498 & -0.69304688 & 2.11215379 \\ 1 & 1.20043369 & -0.69311839 & 2.11215379 \\ 1 & 1.24268149 & 0.71748182 & -1.31838042 \\ 1 & -1.24269823 & 0.71745283 & -1.31838042 \\ 1 & 0.00001674 & -1.43493465 & -1.31838042\end{array}$

AsH3

! Basis set $=B C 6-31 G(d) / 04-S e p-07 / 08: 56: 10$

Nuclear repulsion $=\quad 34.67974186$ ENERGY $($ RB3LYP $)=\quad-2235.53747030$

FORMULA $=$ H3As POINT GROUP $=\mathrm{Cs}$

Cartesian:

$\begin{array}{rrrr}33 & -0.03468852 & 0.06008228 & -0.02326842 \\ 1 & 0.17996146 & -0.31170240 & 1.45879654 \\ 1 & 1.43367057 & -0.28627846 & -0.34546933 \\ 1 & -0.46891086 & -1.38473436 & -0.34546933\end{array}$

AsH3

! Basis set $=B C 6-31 G(d, p) / 04-$ Sep-07/08:56:10

Nuclear repulsion $=\quad 35.24269726$ ENERGY $($ RB3LYP $)=\quad-2235.54903190$

FORMULA $=$ H3AS POINT GROUP $=\mathrm{Cs}$

Cartesian:

$\begin{array}{rrrr}33 & -0.03388400 & 0.05868881 & -0.02279247 \\ 1 & 0.17309351 & -0.29980675 & 1.43764613 \\ 1 & 1.41180291 & -0.27617792 & -0.34274735 \\ 1 & -0.46672436 & -1.36074615 & -0.34274735\end{array}$

AsH3

! Basis set $=$ BC6-31G(d)/04-Sep-07/08:56:10

Nuclear repulsion $=\quad 35.11208924$ ENERGY $($ RHF $)=\quad-2233.70456580$

FORMULA $=$ H3As POINT GROUP $=\mathrm{Cs}$

Cartesian:

$\begin{array}{rrrr}33 & -0.03275745 & 0.05673757 & -0.02212346 \\ 1 & 0.15599500 & -0.27019127 & 1.45367438 \\ 1 & 1.42035201 & -0.24805837 & -0.36180003 \\ 1 & -0.49535115 & -1.35409011 & -0.36180003\end{array}$

AsH3

! Basis set $=B C 6-31 G(d, p) / 04-$ Sep-07/08:56:10 


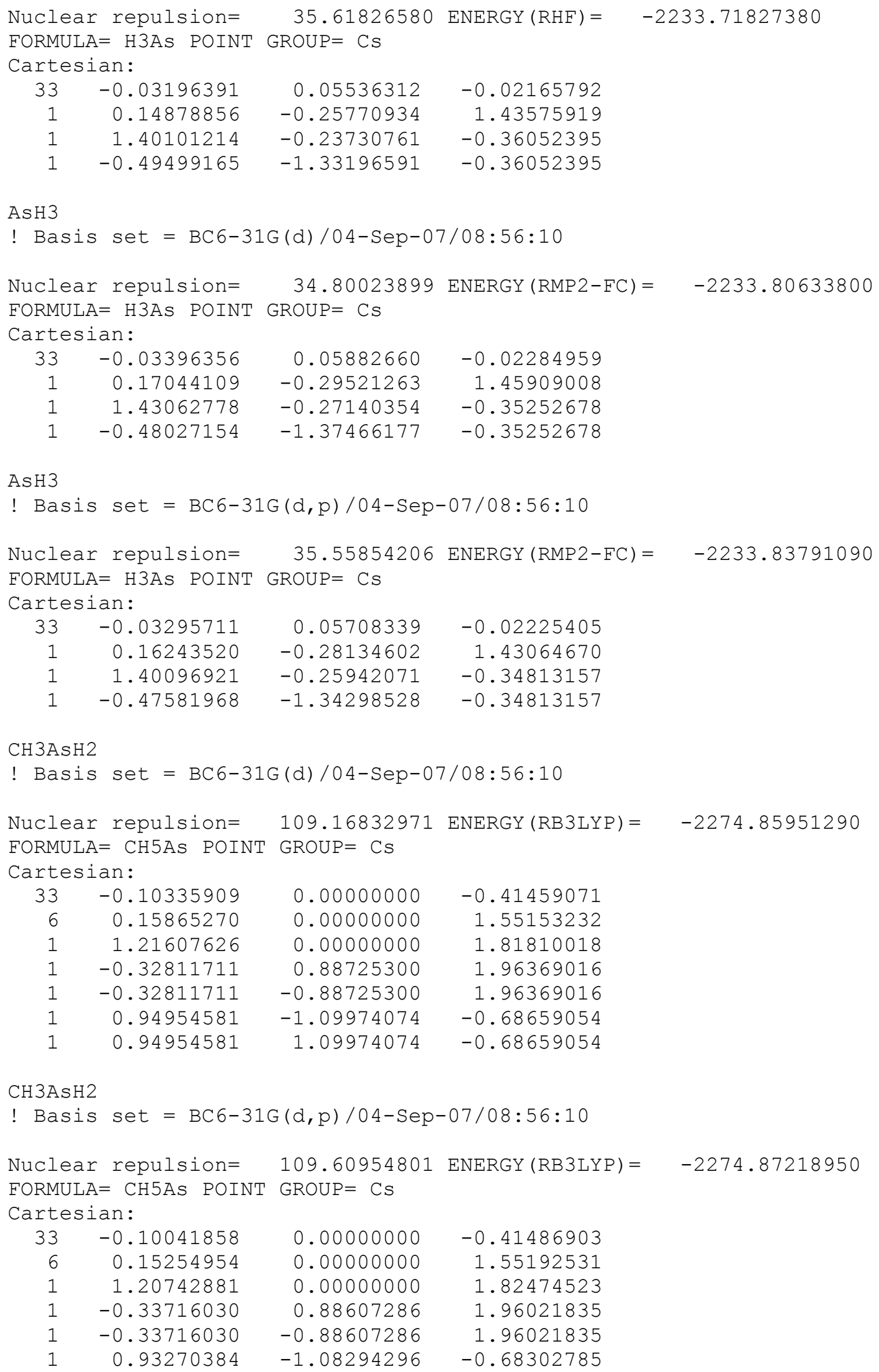




\begin{tabular}{|c|c|c|c|c|}
\hline 1 & 0.93270384 & 1.08294296 & -0.68302785 & \\
\hline \multicolumn{5}{|l|}{ CH3AsH2 } \\
\hline \multicolumn{5}{|c|}{ ! Basis set $=B C 6-31 G(d) / 04-$ Sep-07/08:56:10 } \\
\hline \multirow{2}{*}{ Nuclear } & repulsion $=$ & 110.29994387 & ENERGY $($ RHF $)=$ & -2272.74720200 \\
\hline & $=$ CH5AS POINT & $\mathrm{GROUP}=\mathrm{CS}$ & & \\
\hline \multicolumn{5}{|c|}{ Cartesian: } \\
\hline 33 & -0.09573872 & 0.00000000 & -0.41004089 & \\
\hline 6 & 0.13912633 & 0.00000000 & 1.53831518 & \\
\hline 1 & 1.18329866 & 0.00000000 & 1.81580434 & \\
\hline 1 & -0.34517608 & 0.87769002 & 1.94693352 & \\
\hline 1 & -0.34517608 & -0.87769002 & 1.94693352 & \\
\hline 1 & 0.91583658 & -1.10498616 & -0.70410655 & \\
\hline 1 & 0.91583658 & 1.10498616 & -0.70410655 & \\
\hline \multicolumn{5}{|l|}{ CH3AsH2 } \\
\hline \multicolumn{5}{|l|}{ ! Basis } \\
\hline \multicolumn{2}{|c|}{ Nuclear repulsion= } & 110.67526110 & ENERGY $($ RHF $)=$ & -2272.76191830 \\
\hline \multirow{2}{*}{\multicolumn{5}{|c|}{$\begin{array}{l}\text { FURMULA= CHSAS POINT GROUP= CS } \\
\text { Cartesian: }\end{array}$}} \\
\hline & & & & \\
\hline $33-$ & -0.09282639 & 0.00000000 & -0.41026659 & \\
\hline 6 & 0.13296832 & 0.00000000 & 1.53901311 & \\
\hline 1 & 1.17570155 & 0.00000000 & 1.82258395 & \\
\hline 1 & -0.35478240 & 0.87760133 & 1.94377202 & \\
\hline 1 & -0.35478240 & -0.87760133 & 1.94377202 & \\
\hline 1 & 0.89966211 & -1.09088646 & -0.70270461 & \\
\hline 1 & 0.89966211 & 1.09088646 & -0.70270461 & \\
\hline \multicolumn{5}{|l|}{ CH3AsH2 } \\
\hline \multicolumn{5}{|c|}{ ! Basis } \\
\hline \multicolumn{2}{|c|}{ Nuclear repulsion $=$} & 109.75081787 & ENERGY $($ RMP 2-FC) $=$ & -2272.97959230 \\
\hline & $=$ CH5AS POINT & $\mathrm{GROUP}=\mathrm{Cs}$ & & \\
\hline \multicolumn{5}{|c|}{ Cartesian: } \\
\hline $33-$ & -0.10082792 & 0.00000000 & -0.41154696 & \\
\hline 6 & 0.15245616 & 0.00000000 & 1.54178173 & \\
\hline 1 & 1.20763212 & 0.00000000 & 1.81151529 & \\
\hline 1 & -0.33474053 & 0.88560592 & 1.95210642 & \\
\hline 1 & -0.33474053 & -0.88560592 & 1.95210642 & \\
\hline 1 & 0.93721671 & -1.10344977 & -0.69268438 & \\
\hline 1 & 0.93721671 & 1.10344977 & -0.69268438 & \\
\hline \multicolumn{5}{|c|}{ CH3AsH2 } \\
\hline \multicolumn{5}{|c|}{ ! Basis set $=B C 6-31 G(d, p) / 04-\operatorname{Sep}-07 / 08: 56: 10$} \\
\hline \multirow{2}{*}{\multicolumn{2}{|c|}{$\begin{array}{l}\text { Nuclear repulsion }= \\
\text { FORMULA = CH5As POINT } \\
\text { Cartesian: }\end{array}$}} & 110.42521771 & ENERGY (RMP2-FC) = & -2273.02566190 \\
\hline & & GROUP $=\mathrm{CS}$ & & \\
\hline 33 & -0.09745529 & 0.00000000 & -0.41177764 & \\
\hline 6 & 0.14568500 & 0.00000000 & 1.54099174 & \\
\hline 1 & 1.19492720 & 0.00000000 & 1.81660417 & \\
\hline 1 & -0.34311911 & 0.88173153 & 1.94632491 & \\
\hline 1 & -0.34311911 & -0.88173153 & 1.94632491 & \\
\hline 1 & 0.91661279 & -1.08063882 & -0.68327118 & \\
\hline 1 & 0.91661279 & 1.08063882 & -0.68327118 & \\
\hline
\end{tabular}




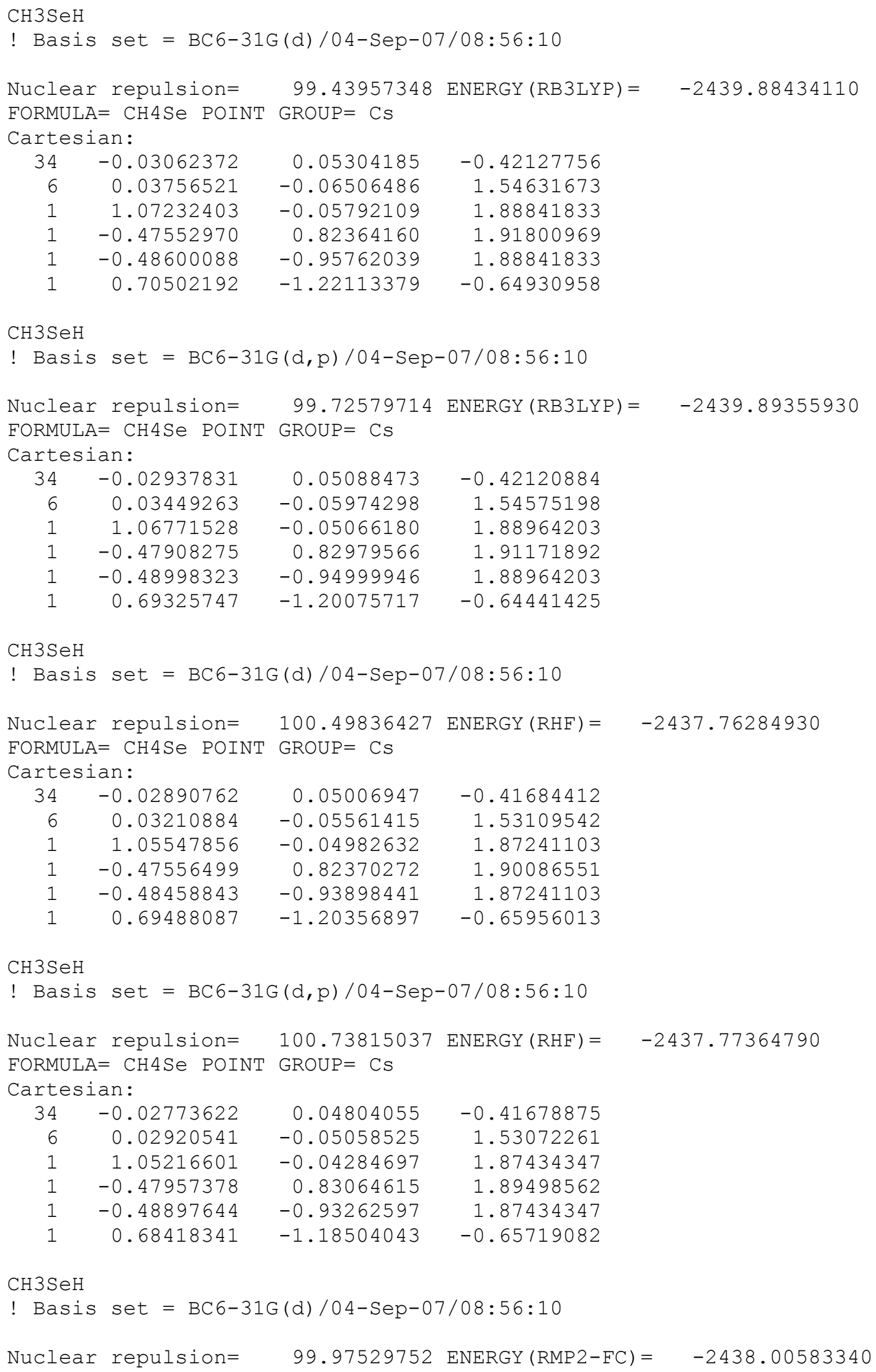




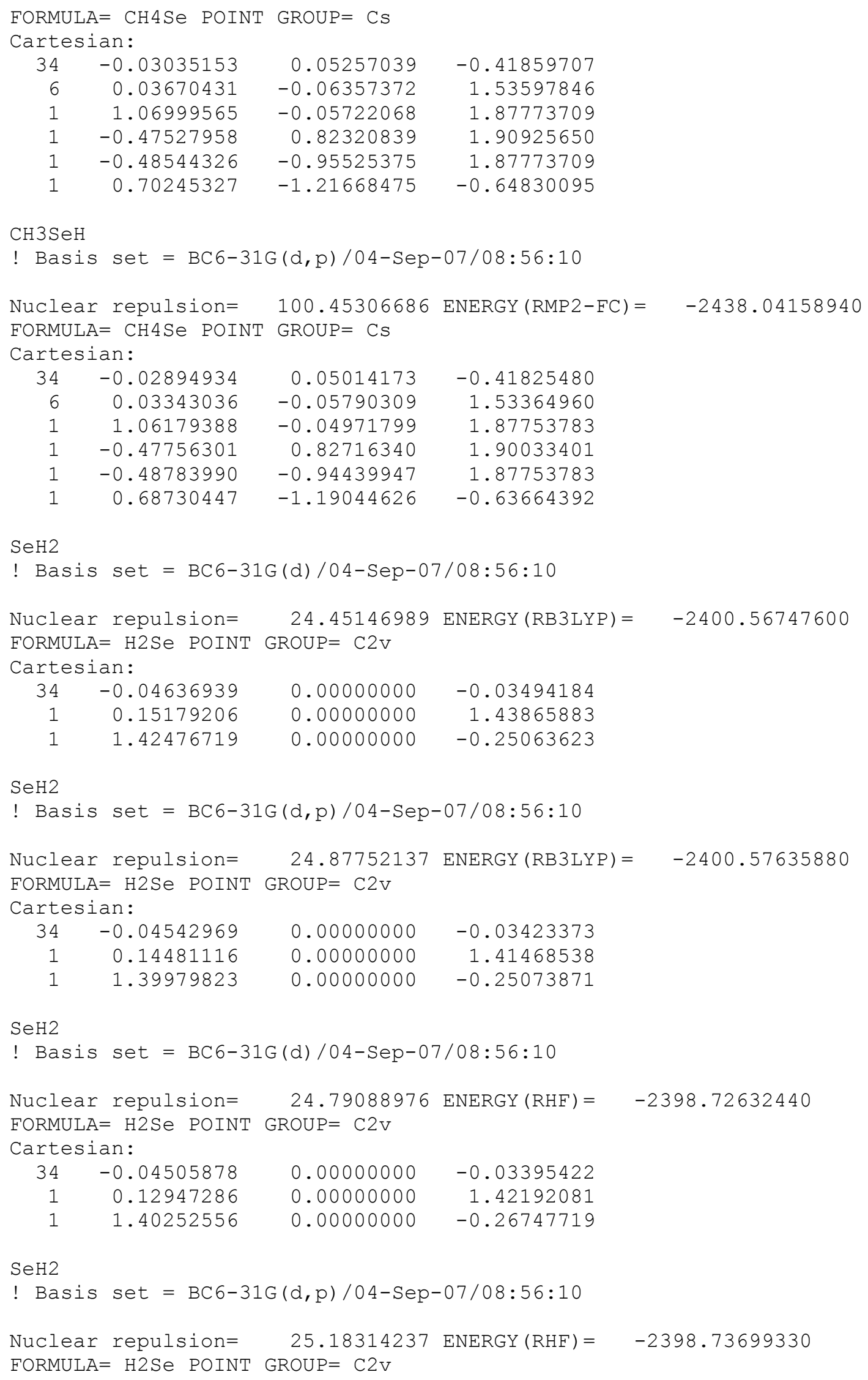




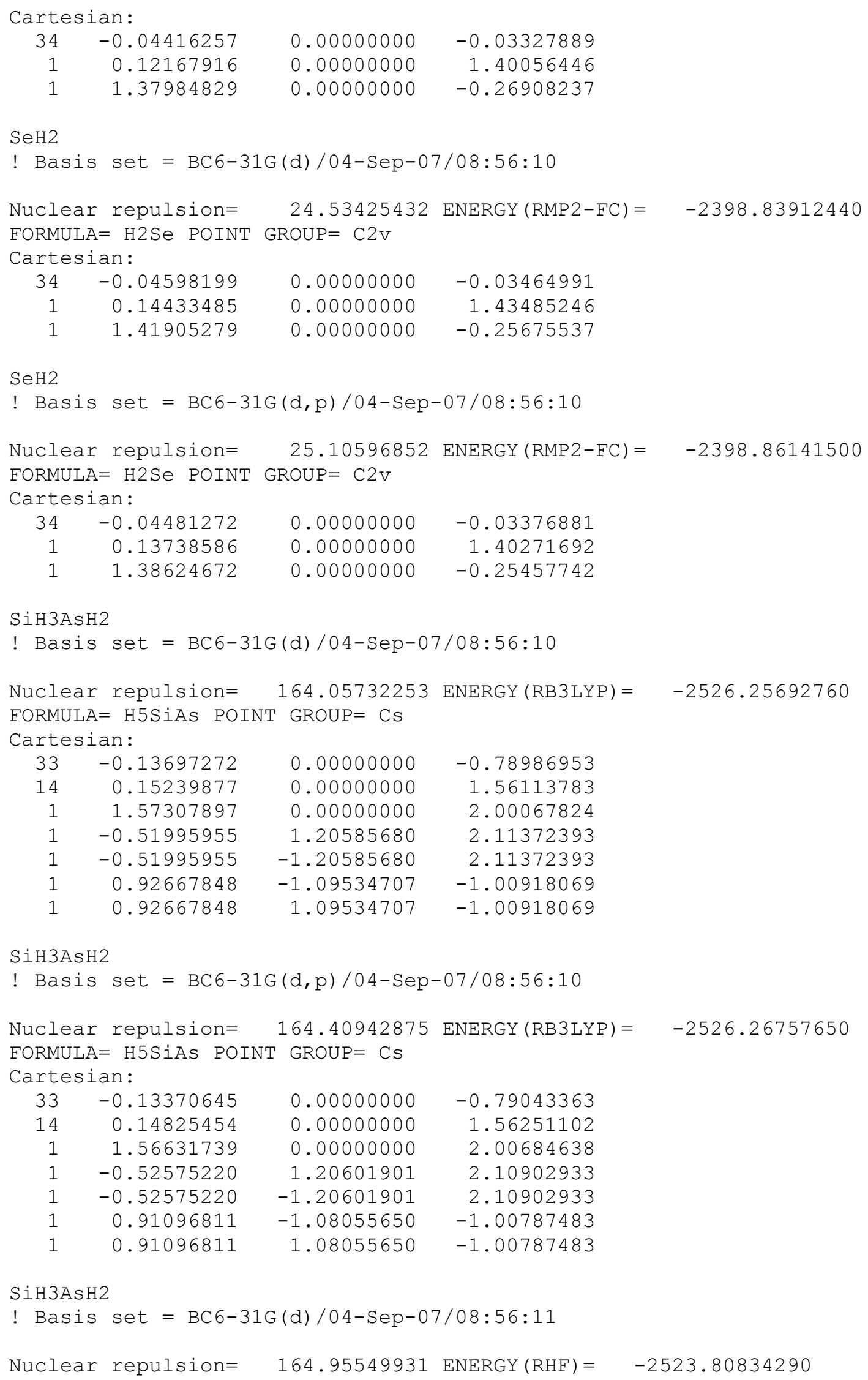




FORMULA = H5SiAs POINT GROUP= CS
$\begin{array}{crrr}\text { Cartesian: } \\ 33 & -0.11549216 & 0.00000000 & -0.78747797 \\ 14 & 0.11386126 & 0.00000000 & 1.56077427 \\ 1 & 1.51893448 & 0.00000000 & 2.00753876 \\ 1 & -0.55995831 & 1.20093034 & 2.08904279 \\ 1 & -0.55995831 & -1.20093034 & 2.08904279 \\ 1 & 0.90908291 & -1.10245036 & -1.02484559 \\ 1 & 0.90908291 & 1.10245036 & -1.02484559\end{array}$

SiH3AsH2

! Basis set $=\operatorname{BC} 6-31 G(d, p) / 04-$ Sep-07/08:56:11

Nuclear repulsion $=\quad 165.23640067$ ENERGY $($ RHF $)=\quad-2523.82130470$ FORMULA $=$ H5SiAs POINT GROUP $=\mathrm{CS}$

Cartesian:

$\begin{array}{rrrr}33 & -0.12484983 & 0.00000000 & -0.78599164 \\ 14 & 0.13483729 & 0.00000000 & 1.56022359 \\ 1 & 1.54600420 & 0.00000000 & 1.99082199 \\ 1 & -0.53320010 & 1.20254046 & 2.09406921 \\ 1 & -0.53320010 & -1.20254046 & 2.09406921 \\ 1 & 0.87635914 & -1.08994606 & -1.04218318 \\ 1 & 0.87635914 & 1.08994606 & -1.04218318\end{array}$

SiH3AsH2

! Basis set $=$ BC6-31G(d)/04-Sep-07/08:56:11

Nuclear repulsion $=\quad 164.75406090$ ENERGY $($ RMP2 - FC $)=\quad-2523.98747090$ FORMULA $=$ H5SiAs POINT GROUP $=\mathrm{Cs}$

Cartesian:

$\begin{array}{rrrr}33 & -0.13353396 & 0.00000000 & -0.78517312 \\ 14 & 0.14710578 & 0.00000000 & 1.55518101 \\ 1 & 1.56708347 & 0.00000000 & 1.98785788 \\ 1 & -0.52506134 & 1.20795708 & 2.09523437 \\ 1 & -0.52506134 & -1.20795708 & 2.09523437 \\ 1 & 0.91508939 & -1.10024751 & -1.02007397 \\ 1 & 0.91508939 & 1.10024751 & -1.02007397\end{array}$

SiH3AsH2

! Basis set $=\operatorname{BC} 6-31 G(d, p) / 04-$ Sep-07/08:56:11

Nuclear repulsion $=\quad 165.43899326$ ENERGY $($ RMP2-FC $)=\quad-2524.03205760$ FORMULA $=$ H5SiAs POINT GROUP $=\mathrm{CS}$ Cartesian:

$\begin{array}{rrrr}33 & -0.12965956 & 0.00000000 & -0.78536304 \\ 14 & 0.14239666 & 0.00000000 & 1.55567323 \\ 1 & 1.55116821 & 0.00000000 & 1.99202288 \\ 1 & -0.52781864 & 1.20089647 & 2.08716775 \\ 1 & -0.52781864 & -1.20089647 & 2.08716775 \\ 1 & 0.89484073 & -1.07939300 & -1.01440161 \\ 1 & 0.89484073 & 1.07939300 & -1.01440161\end{array}$

$\mathrm{SiH} 3 \mathrm{SeH}$

! Basis set $=$ BC6-31G(d)/04-Sep-07/08:56:11

Nuclear repulsion $=\quad 158.61998902$ ENERGY (RB3LYP) $=\quad-2691.29809590$ FORMULA $=$ H4SiSe POINT GROUP $=\mathrm{Cs}$ 


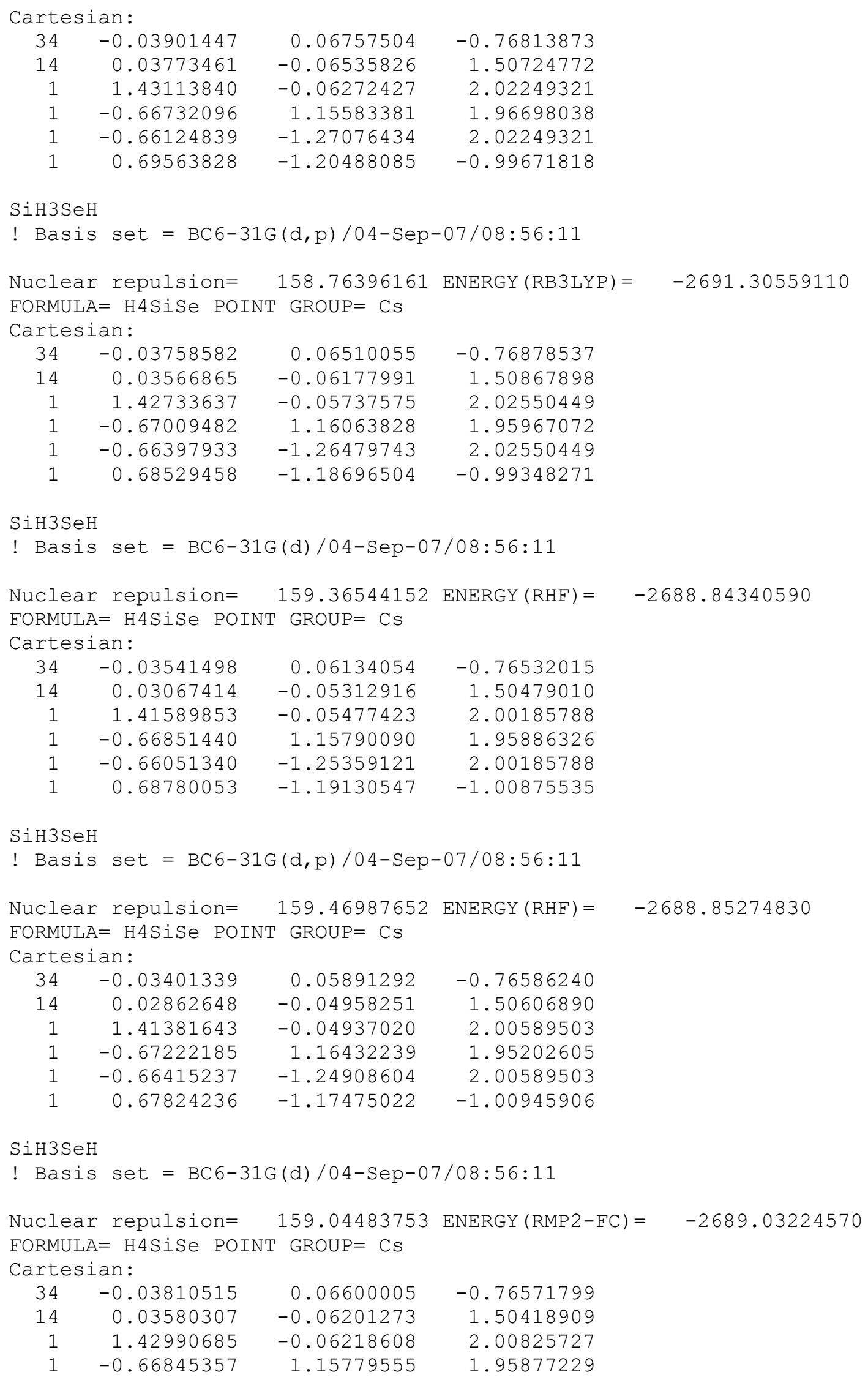




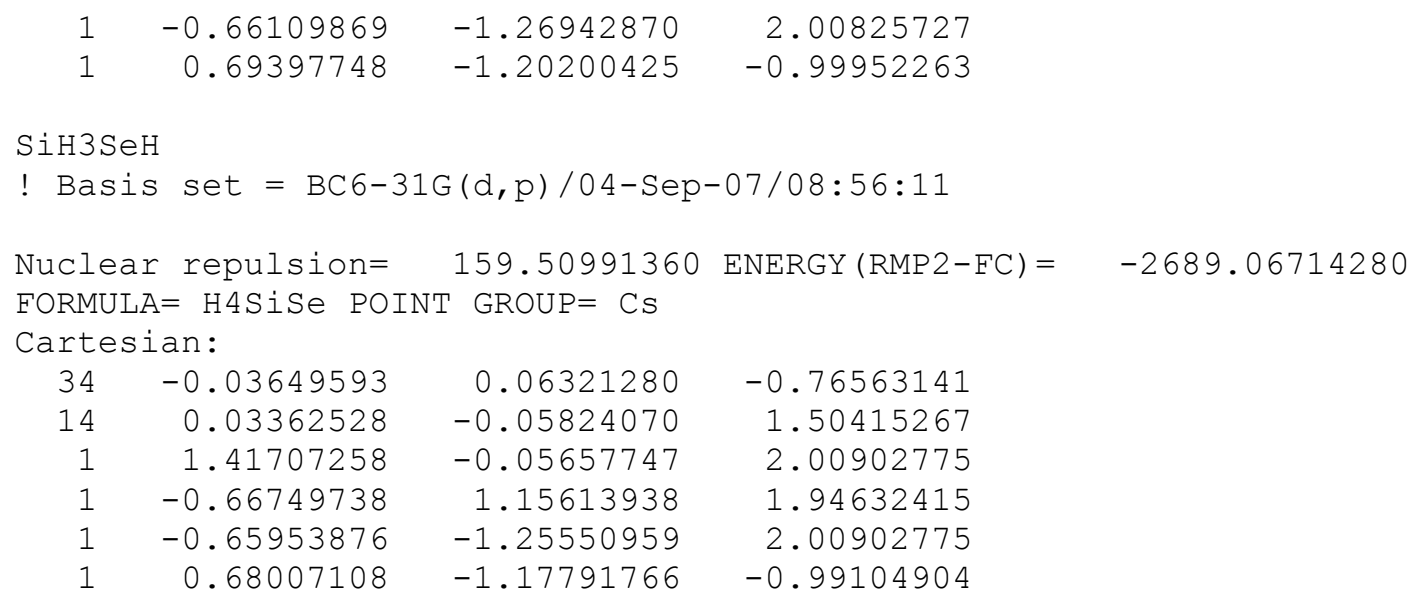

\section{STRUCTURES OPTIMIZED USING STANDARD 6-31G BASIS SET:}

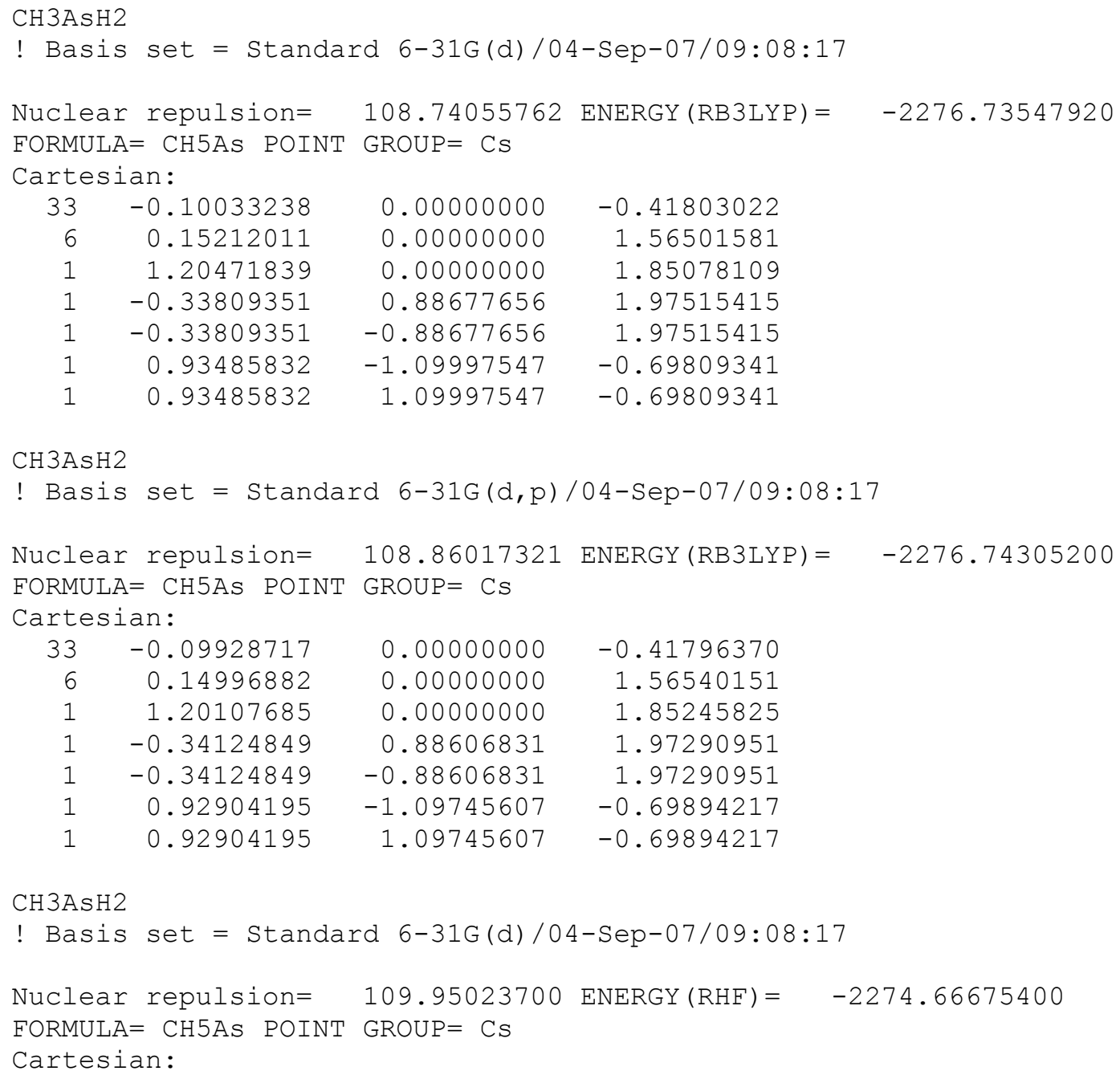




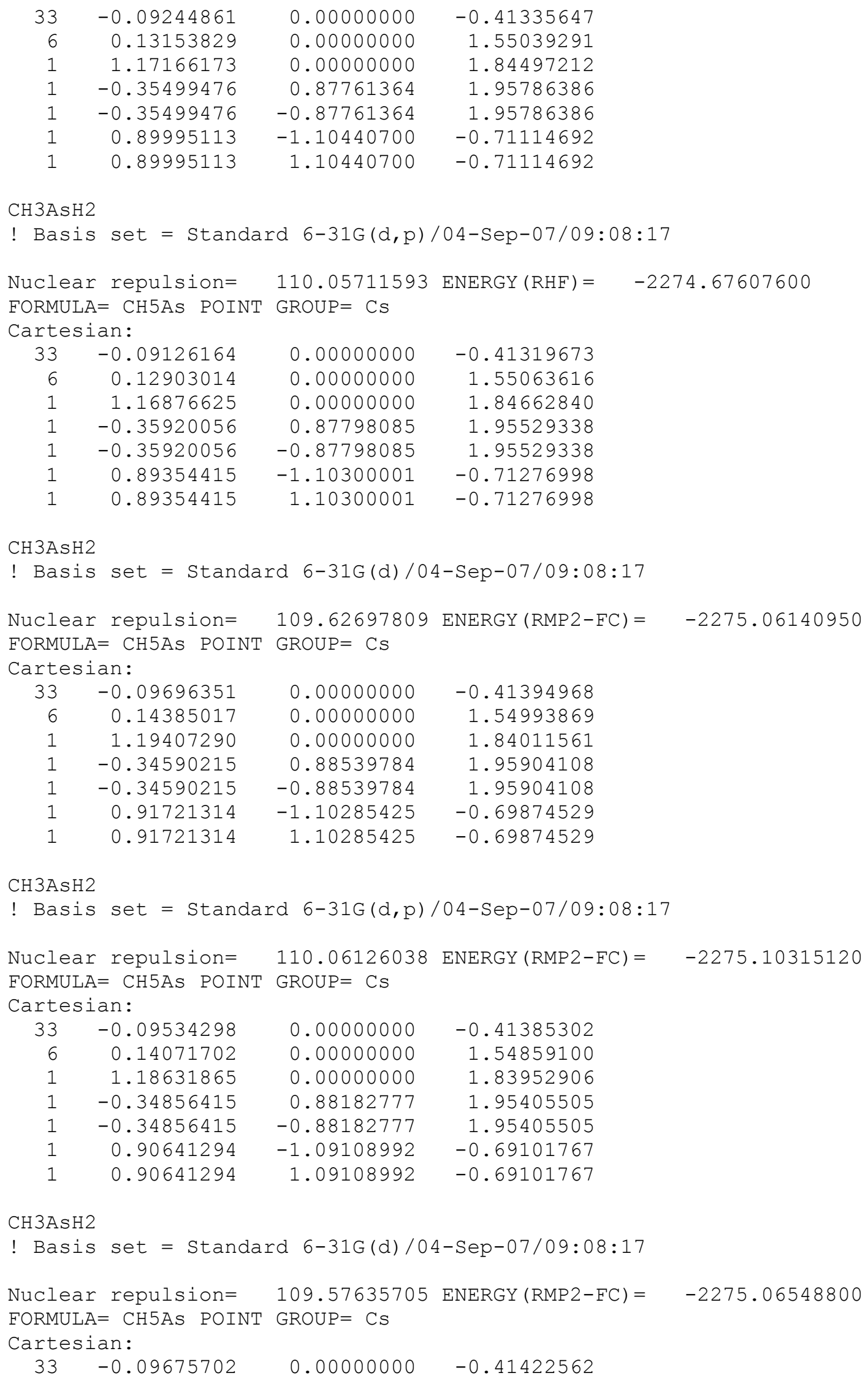




$\begin{array}{rrrr}6 & 0.14362374 & 0.00000000 & 1.55089690 \\ 1 & 1.19430688 & 0.00000000 & 1.84331963 \\ 1 & -0.34718743 & 0.88642368 & 1.95917842 \\ 1 & -0.34718743 & -0.88642368 & 1.95917842 \\ 1 & 0.91565357 & -1.10414263 & -0.69880616 \\ 1 & 0.91565357 & 1.10414263 & -0.69880616\end{array}$

$\mathrm{CH} 3 \mathrm{Br}$

! Basis set = Standard 6-31G(d)/04-Sep-07/09:08:17

Nuclear repulsion $=\quad 88.17224437$ ENERGY $($ RB3LYP $)=\quad-2613.79553640$ FORMULA $=\mathrm{CH} 3 \mathrm{Br}$ POINT GROUP $=\mathrm{C} 3 \mathrm{~V}$

Cartesian:

$0.00000000 \quad 0.00000000$

$6 \quad 0.00000000 \quad 0.00000000$

$-0.42492508$

$1 \quad 1.03678611$

0.00000000

1.54152014

$-0.51839306$

0.89788311

1.87441900

$1-0.51839306$

$-0.89788311$

1.87441900

1.87441900

CH3Br

! Basis set $=$ Standard 6-31G(d,p)/04-Sep-07/09:08:17

Nuclear repulsion $=\quad 88.22016595$ ENERGY $($ RB3LYP $)=\quad-2613.79989290$

FORMULA $=$ CH3Br POINT GROUP $=\mathrm{C} 3 \mathrm{v}$

Cartesian:

$\begin{array}{rrrr}35 & 0.00000000 & 0.00000000 & -0.42467558 \\ 6 & 0.00000000 & 0.00000000 & 1.54112987 \\ 1 & 1.03626593 & 0.00000000 & 1.87228866 \\ 1 & -0.51813297 & 0.89743262 & 1.87228866 \\ 1 & -0.51813297 & -0.89743262 & 1.87228866\end{array}$

$\mathrm{CH} 3 \mathrm{Br}$

! Basis set $=$ Standard 6-31G(d)/04-Sep-07/09:08:17

Nuclear repulsion $=\quad 89.08203025$ ENERGY $($ RHF $)=\quad-2611.71797240$

FORMULA $=\mathrm{CH} 3 \mathrm{Br}$ POINT GROUP $=\mathrm{C} 3 \mathrm{~V}$

Cartesian:

$\begin{array}{rrrr}35 & 0.00000000 & 0.00000000 & -0.42066899 \\ 6 & 0.00000000 & 0.00000000 & 1.52595556 \\ 1 & 1.02502286 & 0.00000000 & 1.85589377 \\ 1 & -0.51251143 & 0.88769584 & 1.85589377 \\ 1 & -0.51251143 & -0.88769584 & 1.85589377\end{array}$

$\mathrm{CH} 3 \mathrm{Br}$

! Basis set = Standard 6-31G(d,p)/04-Sep-07/09:08:17

Nuclear repulsion $=\quad 89.08203028$ ENERGY $($ RHF $)=\quad-2611.72299610$

FORMULA $=$ CH3Br POINT GROUP $=\mathrm{C} 3 \mathrm{v}$

Cartesian:

$\begin{array}{rrrr}35 & 0.00000000 & 0.00000000 & 0.00000000 \\ 6 & 0.00000000 & 0.00000000 & -1.94662455 \\ 1 & 0.00000000 & 1.02502286 & -2.27656276 \\ 1 & 0.88769583 & -0.51251143 & -2.27656276 \\ 1 & -0.88769583 & -0.51251143 & -2.27656276\end{array}$

$\mathrm{CH} 3 \mathrm{Br}$

! Basis set = Standard 6-31G(d)/04-Sep-07/09:08:17 


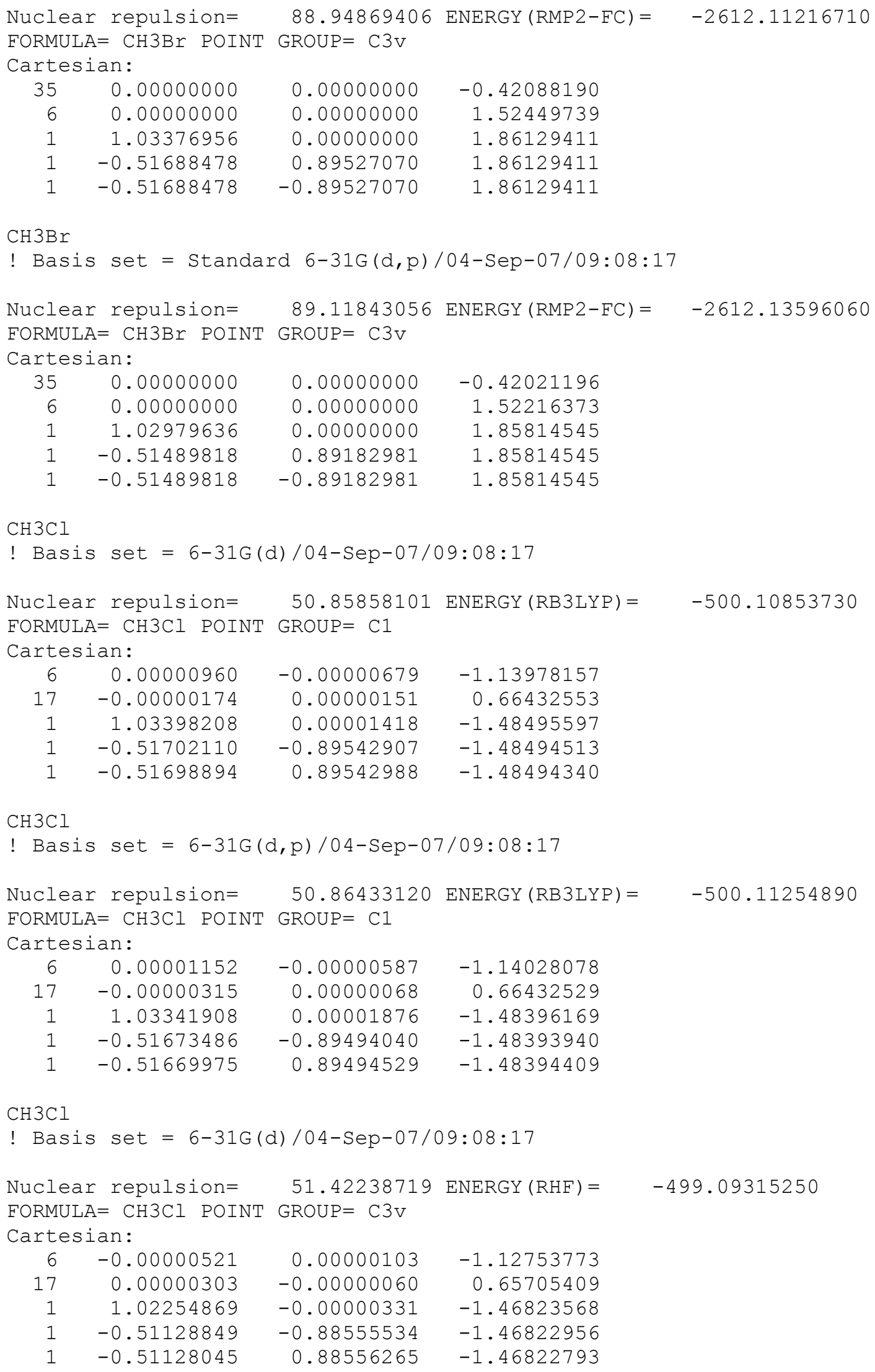




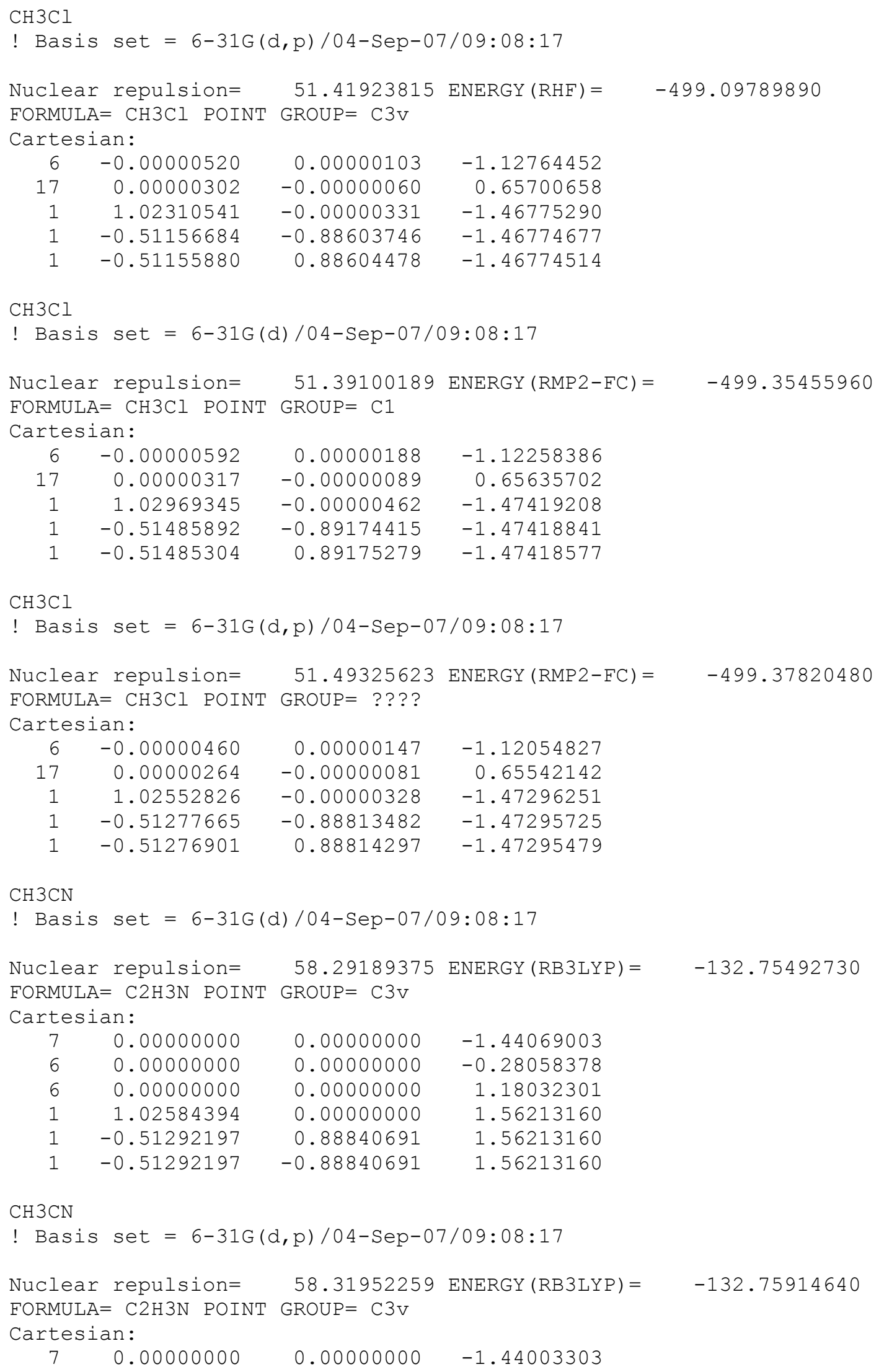

$\begin{array}{rrrr}7 & 0.00000000 & 0.00000000 & -1.44069003 \\ 6 & 0.00000000 & 0.00000000 & -0.28058378 \\ 6 & 0.00000000 & 0.00000000 & 1.18032301 \\ 1 & 1.02584394 & 0.00000000 & 1.56213160 \\ 1 & -0.51292197 & 0.88840691 & 1.56213160 \\ 1 & -0.51292197 & -0.88840691 & 1.56213160\end{array}$

$\mathrm{CH} 3 \mathrm{CN}$

! Basis set $=6-31 G(d, p) / 04-S e p-07 / 09: 08: 17$

Nuclear repulsion $=\quad 58.31952259$ ENERGY $($ RB3LYP $)=\quad-132.75914640$ FORMULA $=\mathrm{C} 2 \mathrm{H} 3 \mathrm{~N}$ POINT GROUP $=\mathrm{C} 3 \mathrm{v}$ 


$\begin{array}{rrrr}6 & 0.00000000 & 0.00000000 & -0.27983018 \\ 6 & 0.00000000 & 0.00000000 & 1.18029698 \\ 1 & 1.02573766 & 0.00000000 & 1.55914347 \\ 1 & -0.51286883 & 0.88831487 & 1.55914347 \\ 1 & -0.51286883 & -0.88831487 & 1.55914347\end{array}$

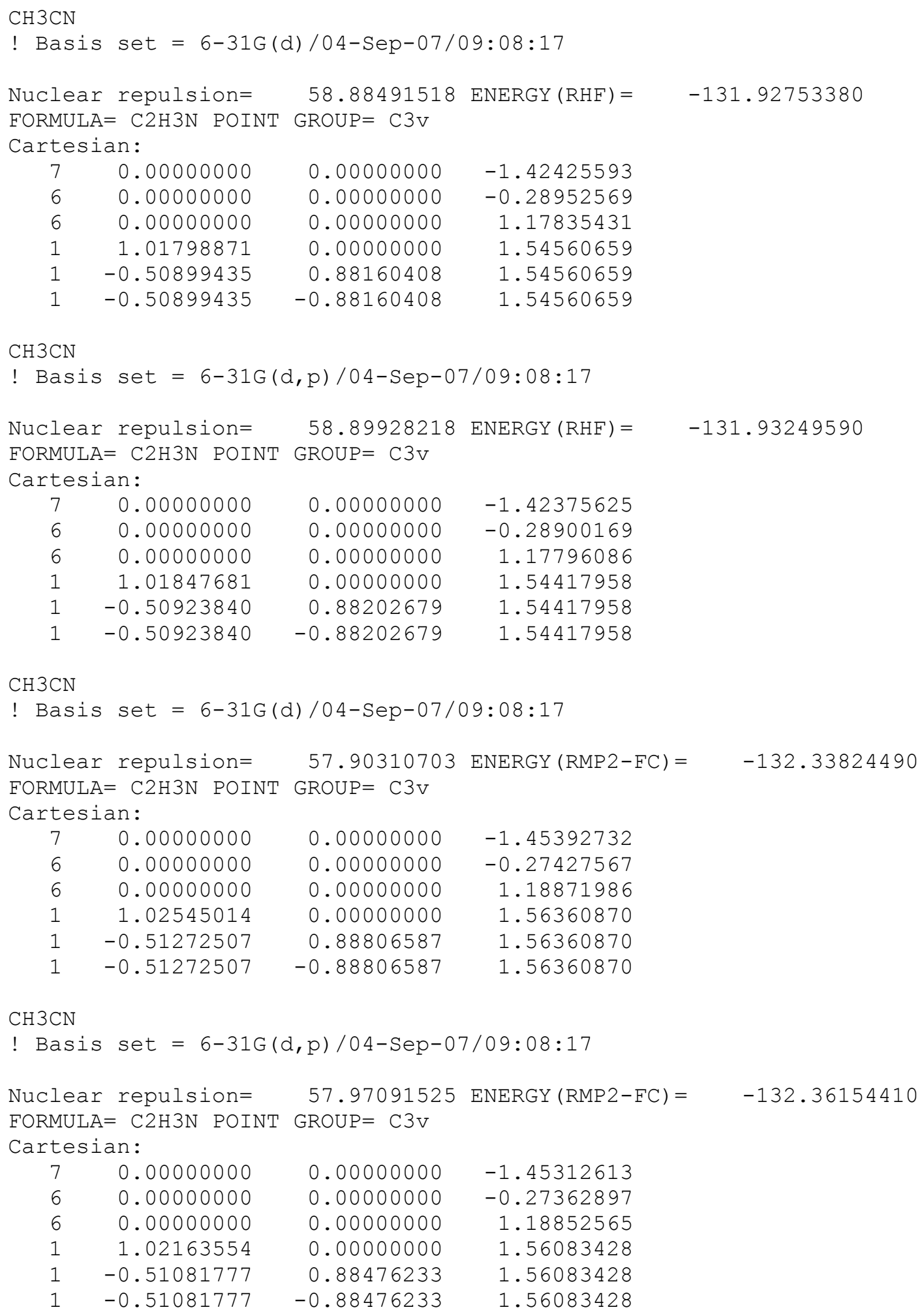




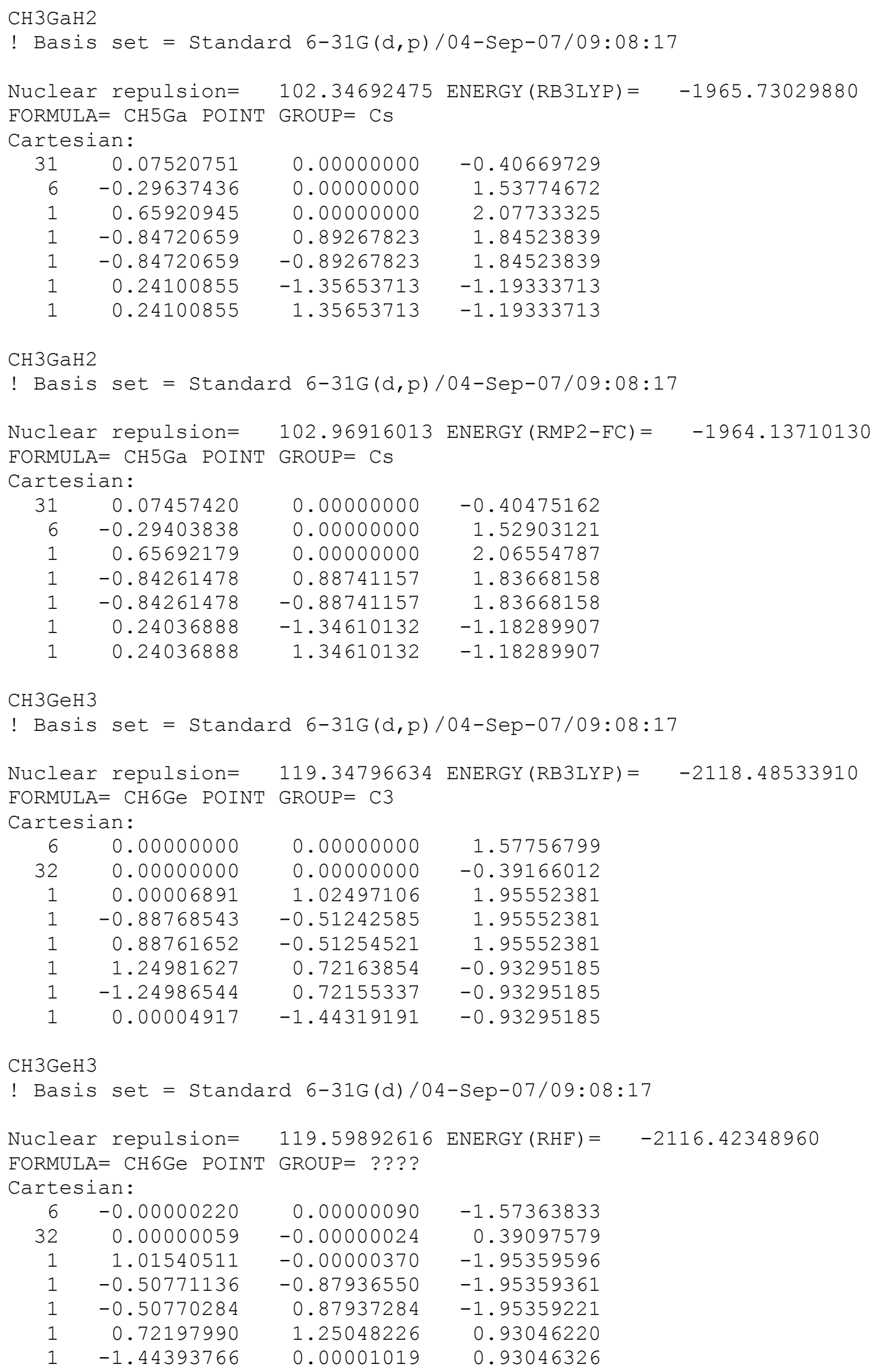




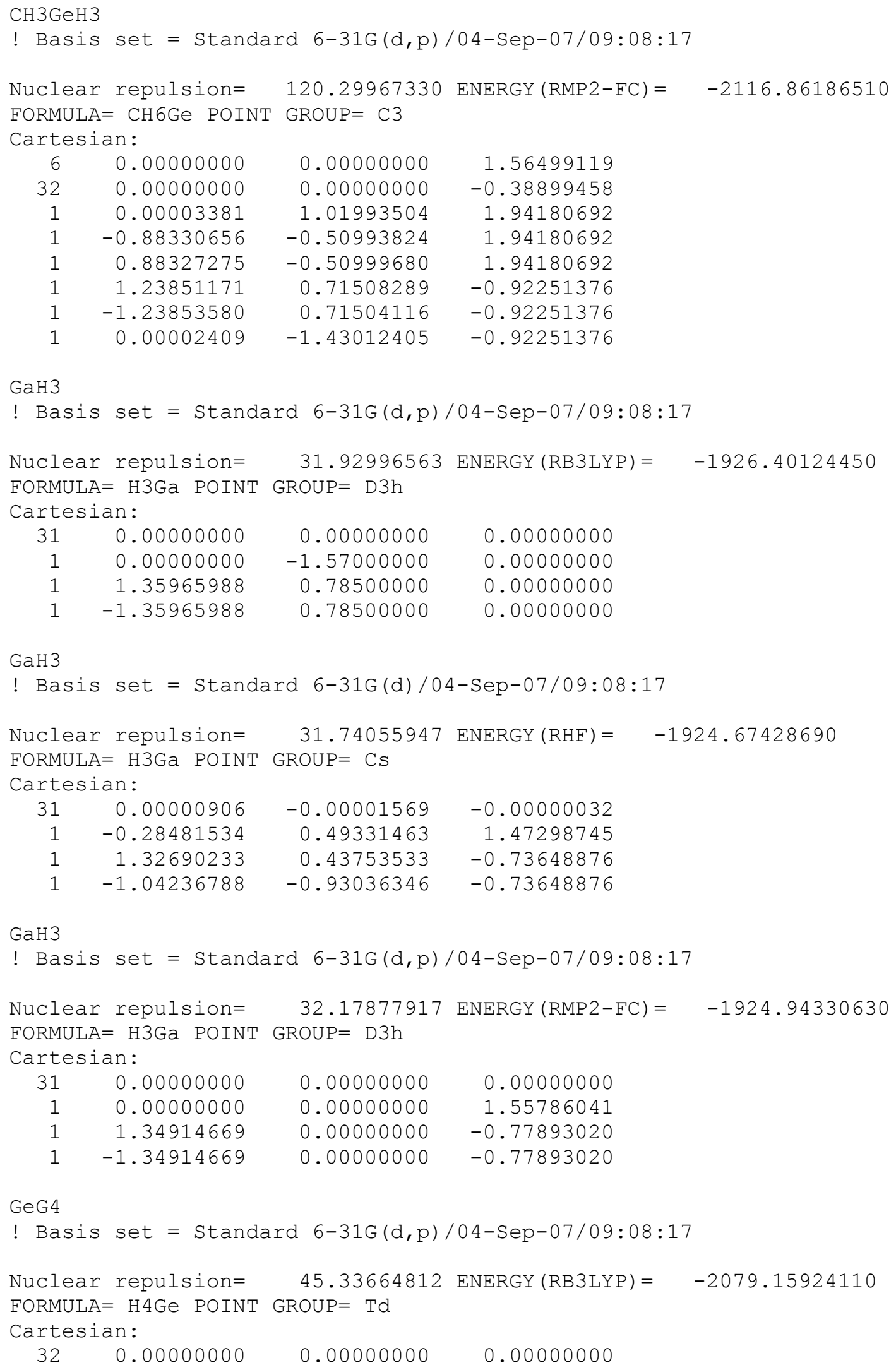




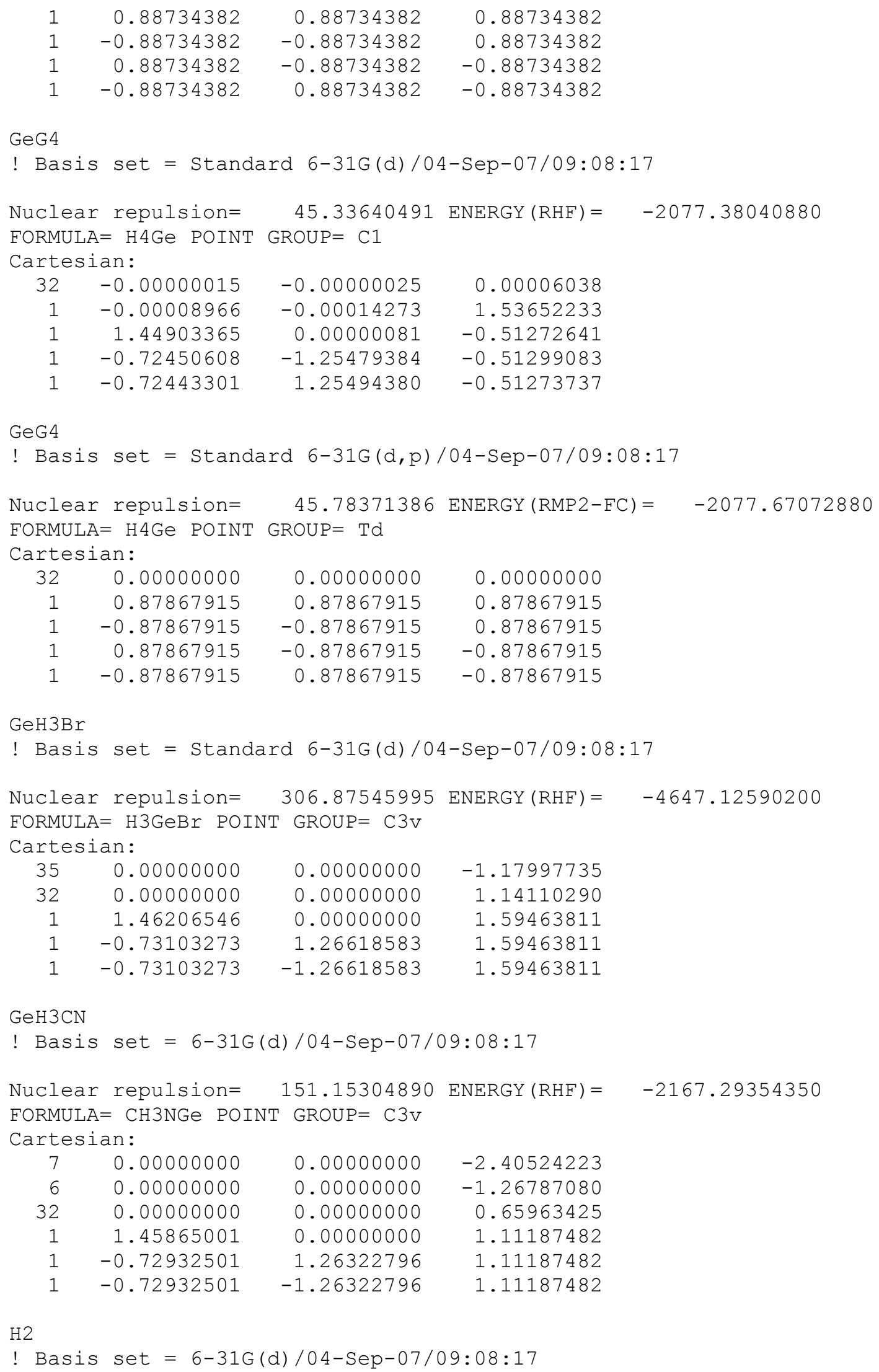




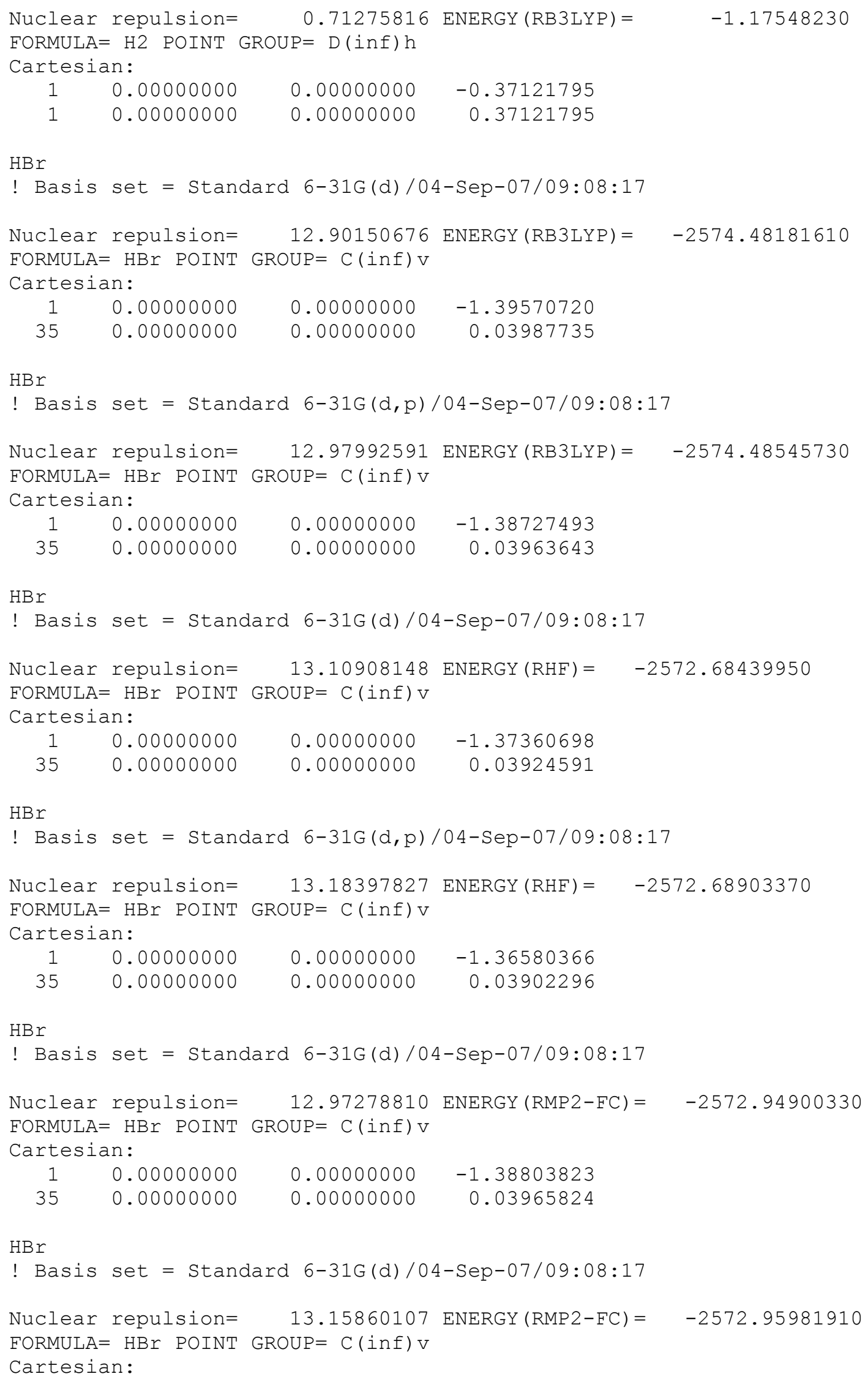




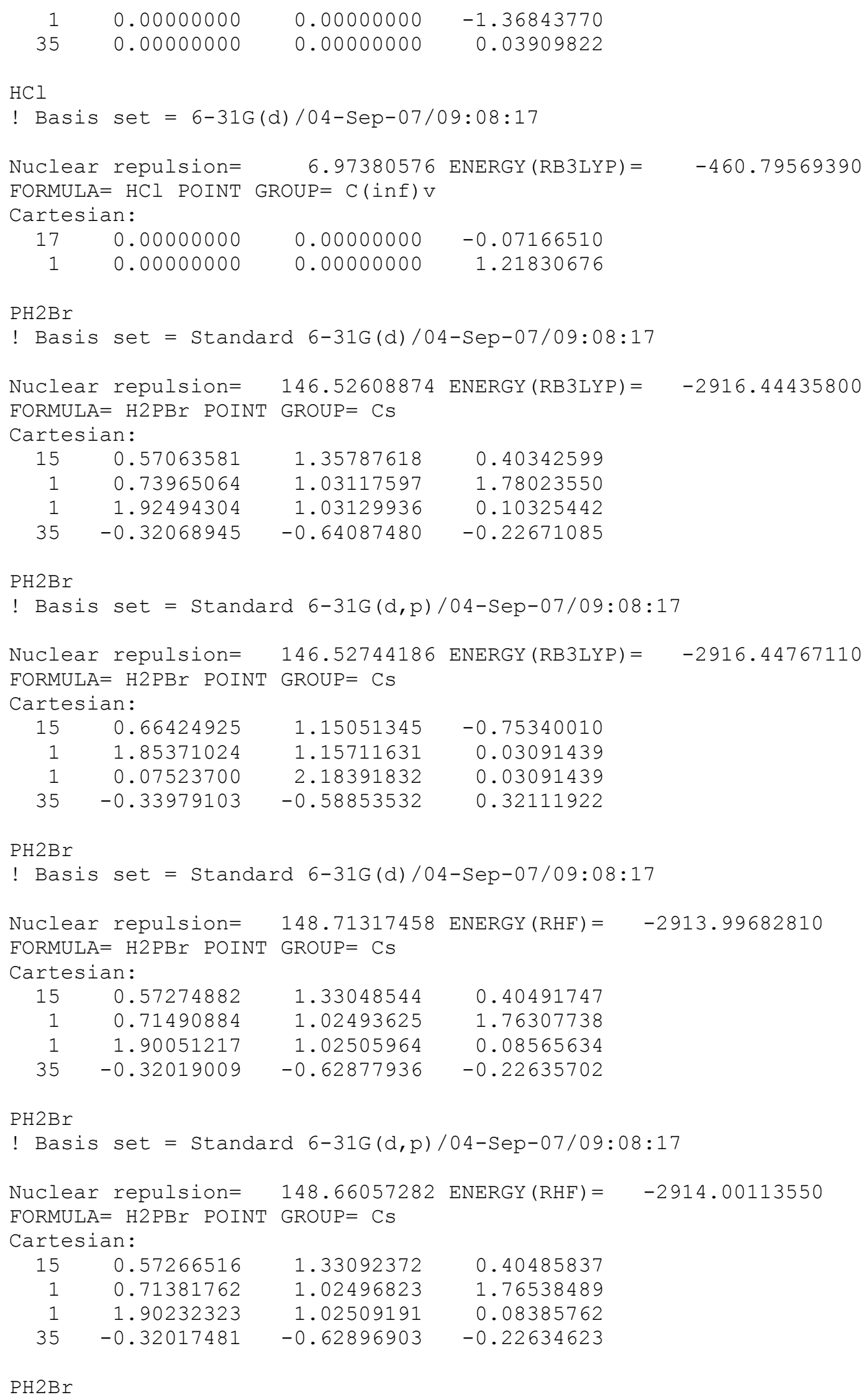


! Basis set = Standard 6-31G(d)/04-Sep-07/09:08:17

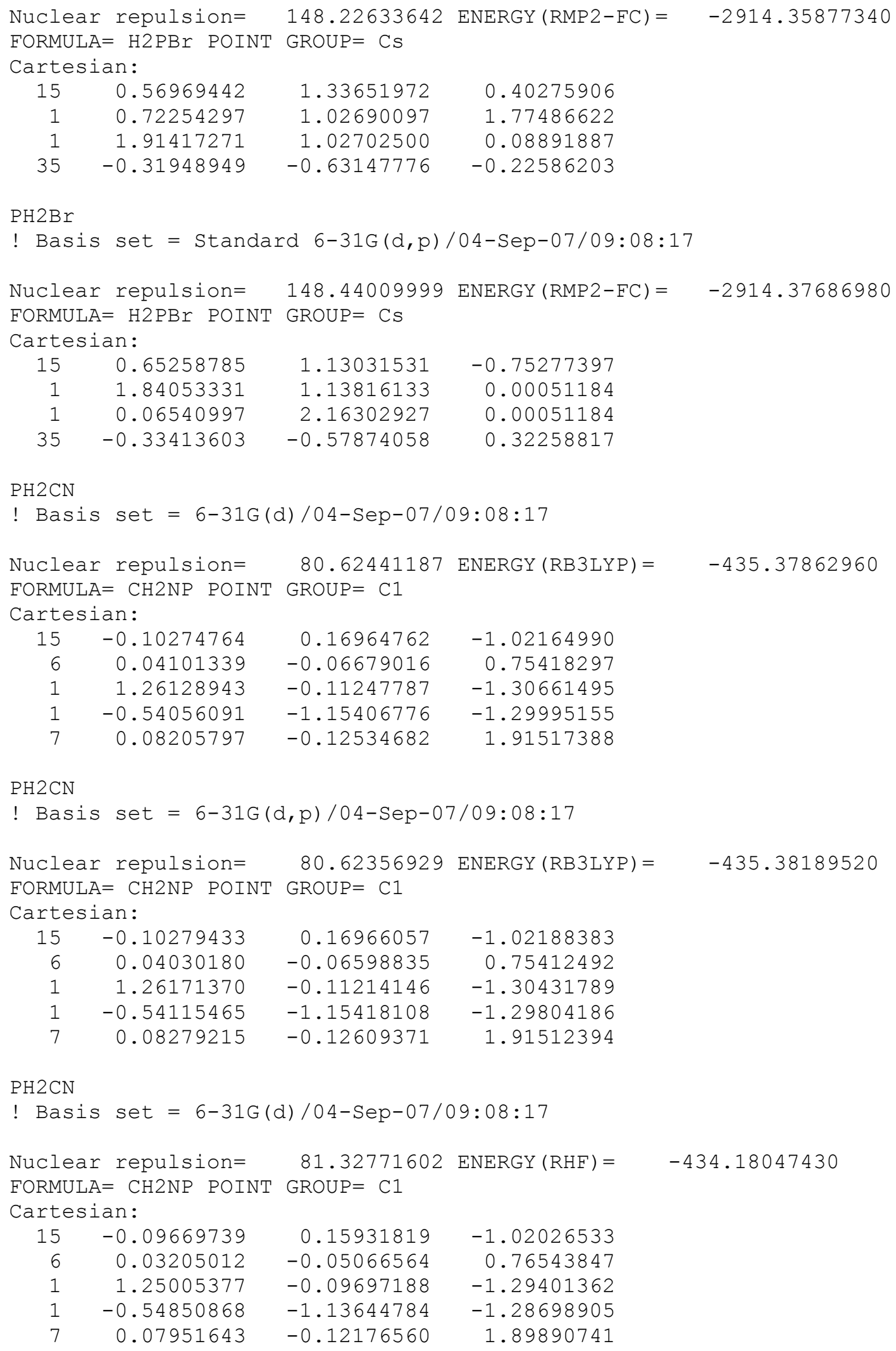




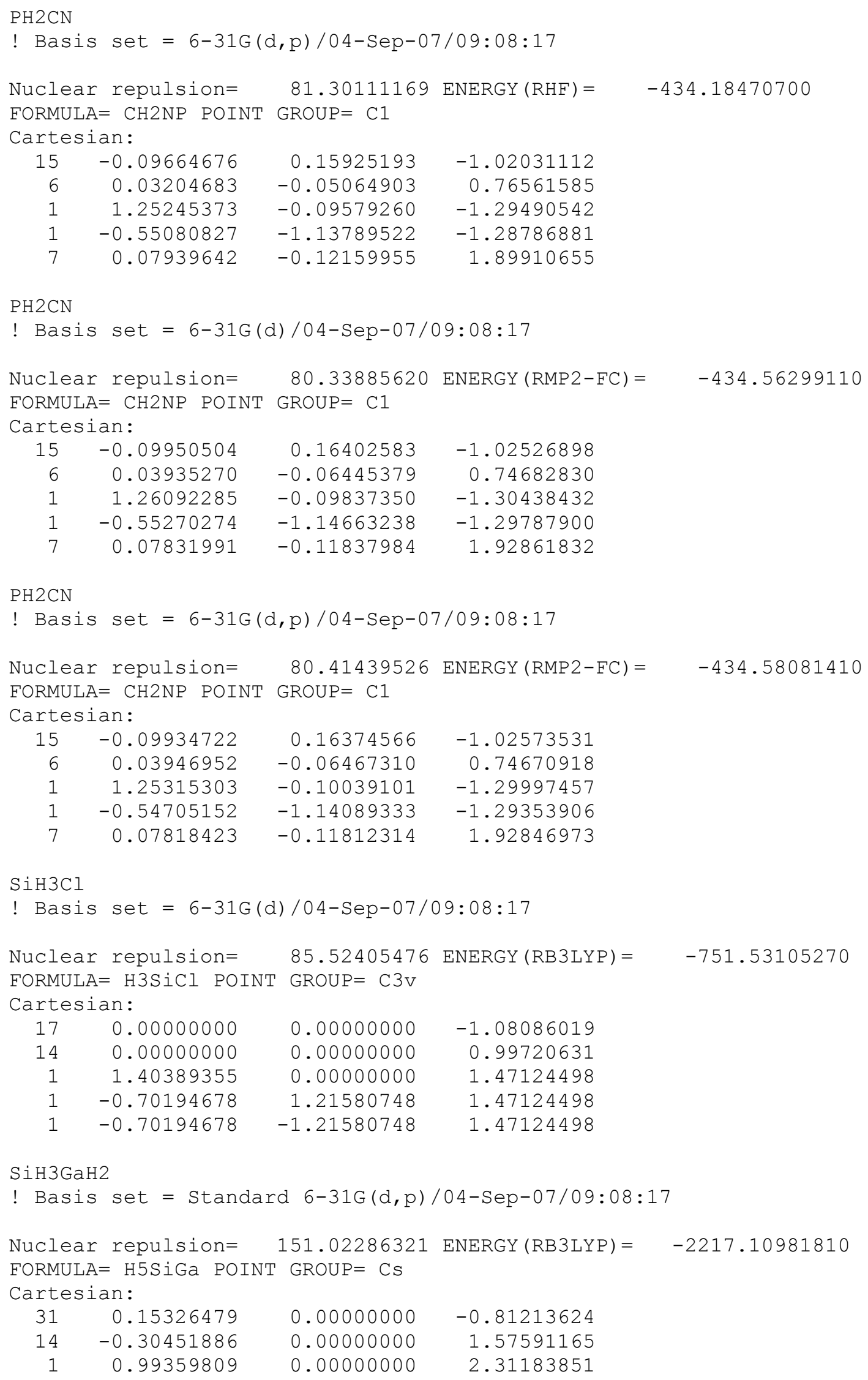




$\begin{array}{rrrr}1 & -1.06374476 & 1.20575236 & 2.00795175 \\ 1 & -1.06374476 & -1.20575236 & 2.00795175 \\ 1 & 0.32297351 & -1.35038938 & -1.60714078 \\ 1 & 0.32297351 & 1.35038938 & -1.60714078\end{array}$

\begin{tabular}{|c|c|c|c|}
\hline \multicolumn{4}{|c|}{$\begin{array}{l}\text { SiH3GaH2 } \\
! \text { Basis }\end{array}$} \\
\hline $\begin{array}{l}\text { Nucle } \\
\text { FORMt } \\
\text { Carte }\end{array}$ & $\begin{array}{l}\text { repulsion= } \\
\mathrm{A}=\mathrm{H} 5 \mathrm{SiGa} \mathrm{PO} \\
\text { ian: }\end{array}$ & $\begin{array}{l}149.98065852 \\
T \text { GROUP }=\mathrm{C} 1\end{array}$ & $\operatorname{ENERGY}(\mathrm{RHF})=$ \\
\hline & -0.00143653 & -0.00248125 & -1.62128742 \\
\hline & -0.00040860 & -0.00071127 & 0.83435961 \\
\hline & 1.38631924 & 0.01189006 & -2.13884137 \\
\hline & -0.68414672 & -1.18496830 & -2.19007887 \\
\hline & -0.68287103 & 1.20653689 & -2.13883632 \\
\hline & -0.67207818 & -1.16408061 & 1.67340255 \\
\hline & 0.68555479 & 1.18740875 & 1.6272301 \\
\hline
\end{tabular}

SiH3GaH2

! Basis set $=$ Standard 6-31G $(\mathrm{d}, \mathrm{p}) / 04-\mathrm{Sep}-07 / 09: 08: 17$

Nuclear repulsion $=\quad 151.85376860$ ENERGY $($ RMP2 - FC $)=\quad-2215.12501470$ FORMULA $=$ H5SiGa POINT GROUP $=$ CS

Cartesian:

$\begin{array}{rrrr}31 & 0.15172703 & 0.00000000 & -0.80866524 \\ 14 & -0.30069750 & 0.00000000 & 1.56988614 \\ 1 & 0.98691140 & 0.00000000 & 2.30126638 \\ 1 & -1.05774326 & 1.19780222 & 1.98978347 \\ 1 & -1.05774326 & -1.19780222 & 1.98978347 \\ 1 & 0.31740103 & -1.33996318 & -1.59530834 \\ 1 & 0.31740103 & 1.33996318 & -1.59530834\end{array}$

SiH3GeH3

! Basis set = Standard 6-31G $(\mathrm{d}, \mathrm{p}) / 04-\mathrm{Sep}-07 / 09: 08: 17$

Nuclear repulsion $=\quad 171.76106638$ ENERGY $($ RB3LYP $)=\quad-2369.86639390$ FORMULA $=$ H6SiGe POINT GROUP $=\mathrm{C} 3$

Cartesian:

$\begin{array}{rrrr}14 & 0.00000000 & 0.00000000 & 1.61625413 \\ 32 & 0.00000000 & 0.00000000 & -0.78248147 \\ 1 & 0.00004209 & 1.39449894 & 2.13302199 \\ 1 & -1.20769255 & -0.69721302 & 2.13302199 \\ 1 & 1.20765046 & -0.69728592 & 2.13302199 \\ 1 & 1.24679547 & 0.71985775 & -1.32907224 \\ 1 & -1.24681283 & 0.71982767 & -1.32907224 \\ 1 & 0.00001737 & -1.43968542 & -1.32907224\end{array}$

SiH3GeH3

! Basis set = Standard 6-31G(d)/04-Sep-07/09:08:17

Nuclear repulsion $=\quad 171.60200729$ ENERGY $($ RHF $)=\quad-2367.46775200$ FORMULA $=$ H6SiGe POINT GROUP $=\mathrm{C} 3$

Cartesian:

$\begin{array}{rrrr}14 & -0.00000032 & 0.00000049 & -1.62064249 \\ 32 & 0.00000016 & -0.00000024 & 0.78453679 \\ 1 & 1.38554961 & -0.00000206 & -2.13287436\end{array}$




$\begin{array}{rrrr}1 & -0.69277786 & -1.19991944 & -2.13287435 \\ 1 & -0.69277308 & 1.19992362 & -2.13287340 \\ 1 & 0.72062180 & 1.24815266 & 1.32748008 \\ 1 & -1.44124276 & -0.00000029 & 1.32748015 \\ 1 & 0.72062167 & -1.24815347 & 1.32747953\end{array}$

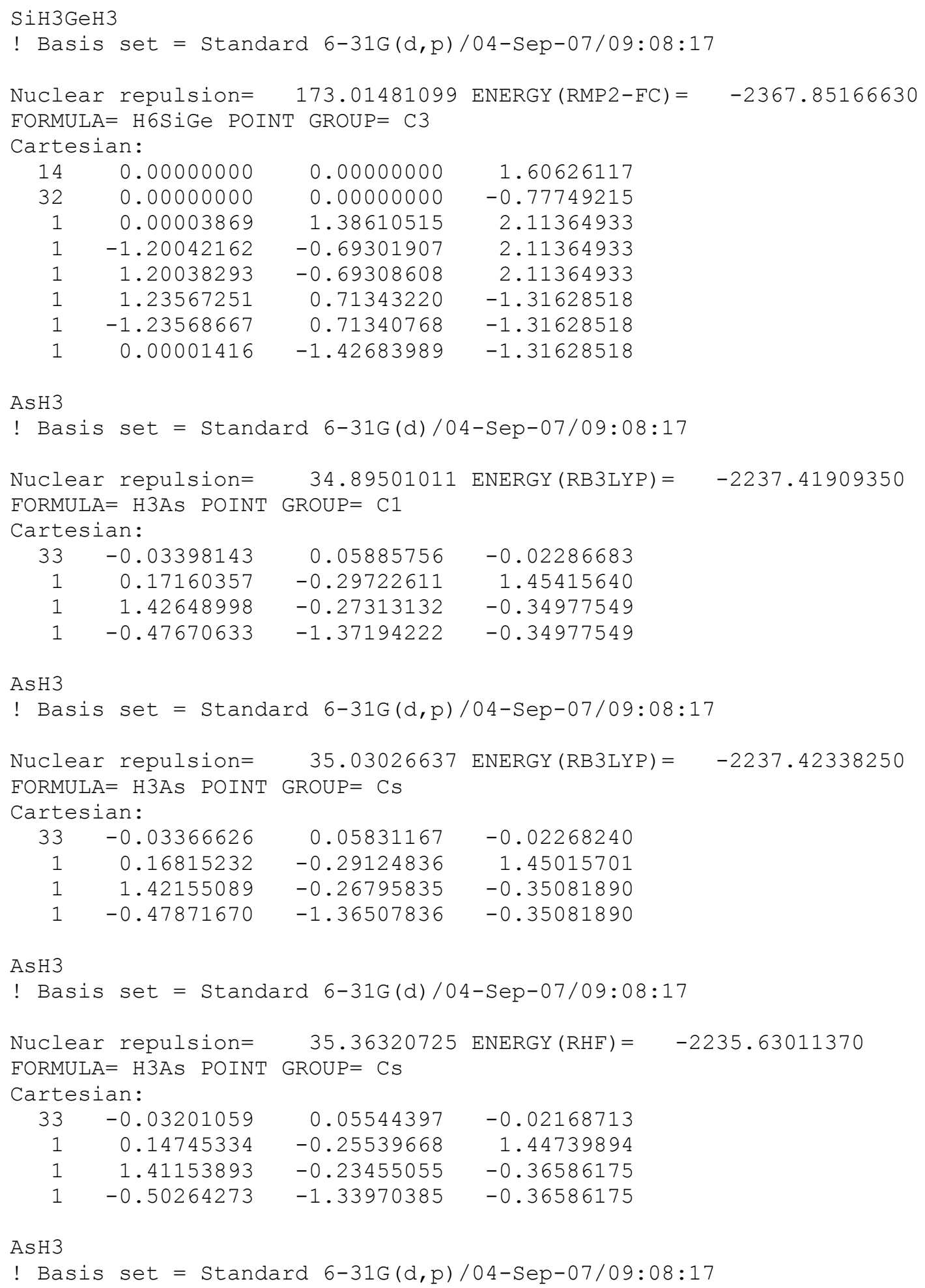




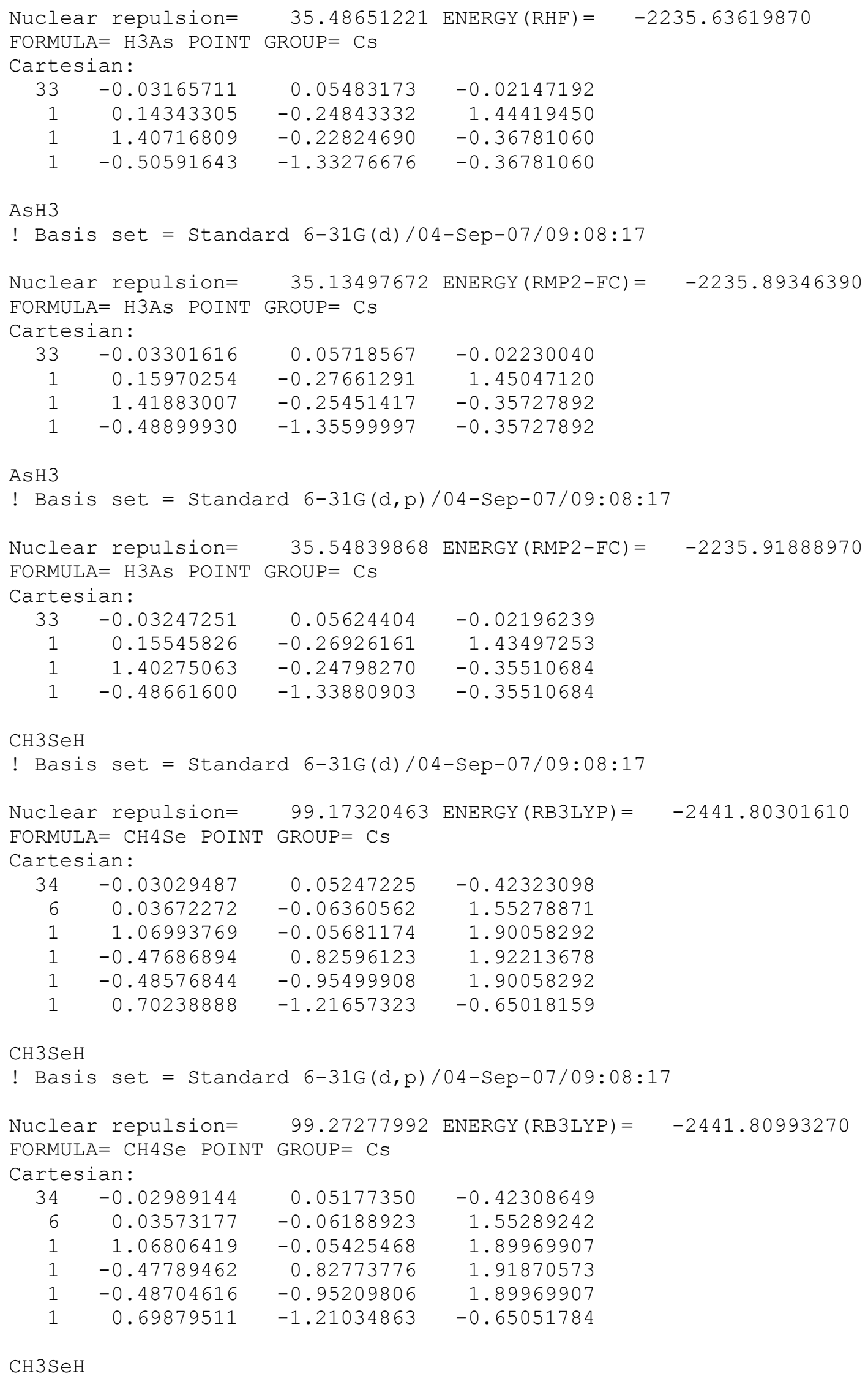


! Basis set = Standard 6-31G(d)/04-Sep-07/09:08:18

Nuclear repulsion $=\quad 100.31674807$ ENERGY $($ RHF $)=\quad-2439.72681810$

FORMULA $=\mathrm{CH} 4 \mathrm{Se}$ POINT GROUP $=\mathrm{Cs}$

Cartesian:

$\begin{array}{rrrr}34 & -0.02843235 & 0.04924627 & -0.41845629 \\ 6 & 0.03086692 & -0.05346307 & 1.53595310 \\ 1 & 1.05242790 & -0.04790709 & 1.88393458 \\ 1 & -0.47721257 & 0.82655642 & 1.90403749 \\ 1 & -0.48472520 & -0.93538284 & 1.88393458 \\ 1 & 0.69100811 & -1.19686115 & -0.66011130\end{array}$

$\mathrm{CH} 3 \mathrm{SeH}$

! Basis set $=$ Standard 6-31G $(\mathrm{d}, \mathrm{p}) / 04-\mathrm{Sep}-07 / 09: 08: 18$

Nuclear repulsion $=\quad 100.39861492$ ENERGY $($ RHF $)=\quad-2439.73512420$

FORMULA $=$ CH4Se POINT GROUP $=\mathrm{CS}$

Cartesian:

$\begin{array}{rrrr}34 & -0.02802342 & 0.04853799 & -0.41827623 \\ 6 & 0.02984458 & -0.05169233 & 1.53594159 \\ 1 & 1.05166118 & -0.04524768 & 1.88350490 \\ 1 & -0.47890907 & 0.82949484 & 1.90064015 \\ 1 & -0.48664495 & -0.93338914 & 1.88350490 \\ 1 & 0.68762164 & -1.19099562 & -0.66190774\end{array}$

$\mathrm{CH} 3 \mathrm{SeH}$

! Basis set = Standard 6-31G(d)/04-Sep-07/09:08:18

Nuclear repulsion $=\quad 99.97581002$ ENERGY $($ RMP2-FC $)=\quad-2440.12395790$ FORMULA $=$ CH4Se POINT GROUP $=\mathrm{Cs}$

Cartesian:

$\begin{array}{rrrr}34 & -0.02999431 & 0.05195168 & -0.41937736 \\ 6 & 0.03592845 & -0.06222990 & 1.53686414 \\ 1 & 1.06713962 & -0.05640402 & 1.88604253 \\ 1 & -0.47611930 & 0.82466282 & 1.91011260 \\ 1 & -0.48472250 & -0.95237203 & 1.88604253 \\ 1 & 0.69793815 & -1.20886434 & -0.64455237\end{array}$

$\mathrm{CH} 3 \mathrm{SeH}$

! Basis set = Standard 6-31G(d)/04-Sep-07/09:08:18

Nuclear repulsion $=\quad 100.30440013$ ENERGY $($ RMP2-FC $)=\quad-2440.15784900$

FORMULA $=\mathrm{CH} 4 \mathrm{Se}$ POINT GROUP $=\mathrm{Cs}$

Cartesian:

$\begin{array}{rrrr}34 & -0.02933857 & 0.05081589 & -0.41898104 \\ 6 & 0.03452991 & -0.05980756 & 1.53489615 \\ 1 & 1.06137517 & -0.05321592 & 1.88378767 \\ 1 & -0.47605716 & 0.82455518 & 1.90389489 \\ 1 & -0.48460125 & -0.94578581 & 1.88378767 \\ 1 & 0.68961509 & -1.19444838 & -0.63549185\end{array}$

$\mathrm{SeH} 2$

! Basis set $=$ Standard 6-31G(d)/04-Sep-07/09:08:18

Nuclear repulsion $=\quad 24.54653226$ ENERGY $($ RB3LYP $)=\quad-2402.48953590$ FORMULA $=\mathrm{H} 2 \mathrm{Se}$ POINT GROUP $=\mathrm{C} 2 \mathrm{~V}$

Cartesian: 


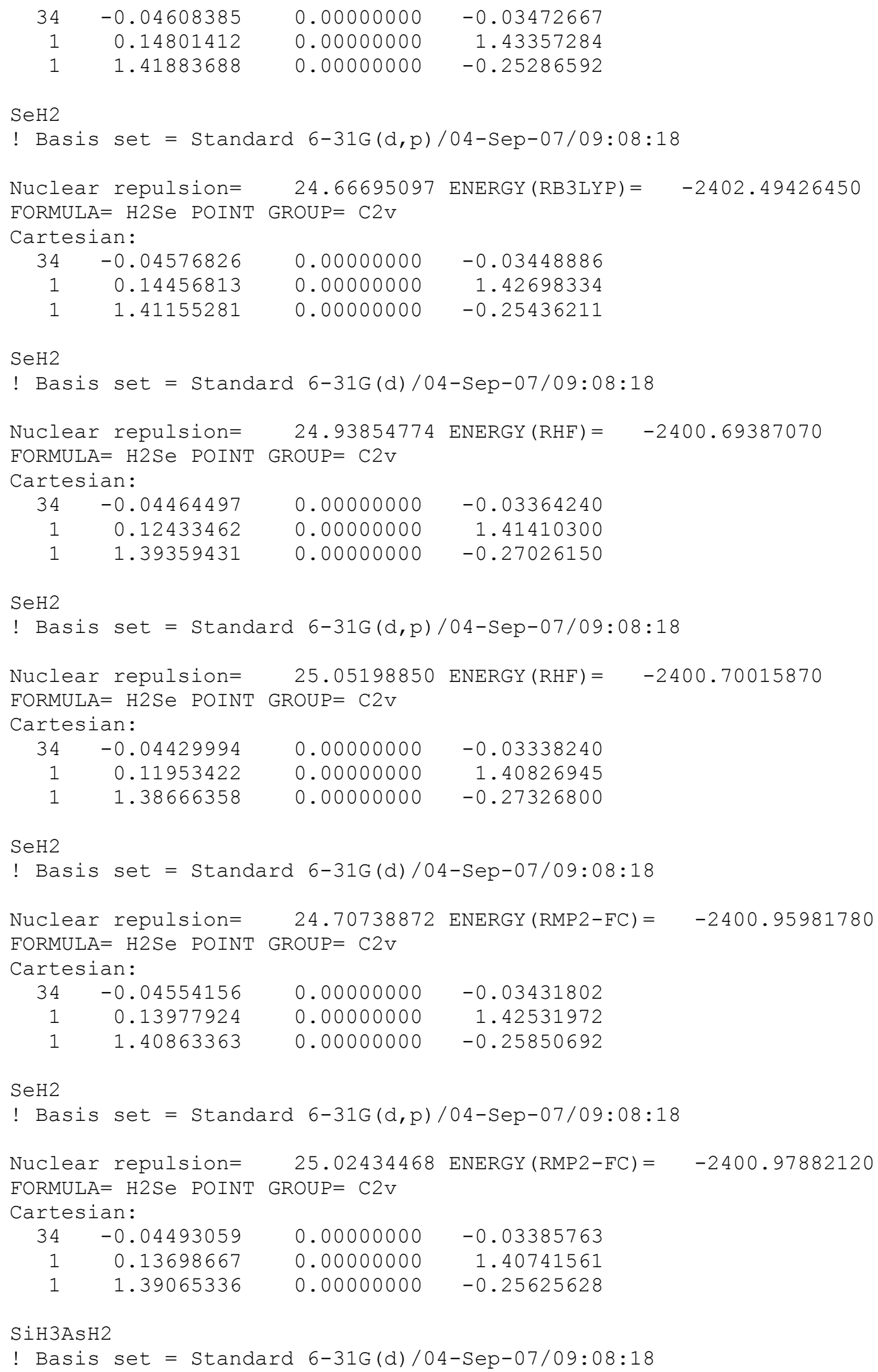




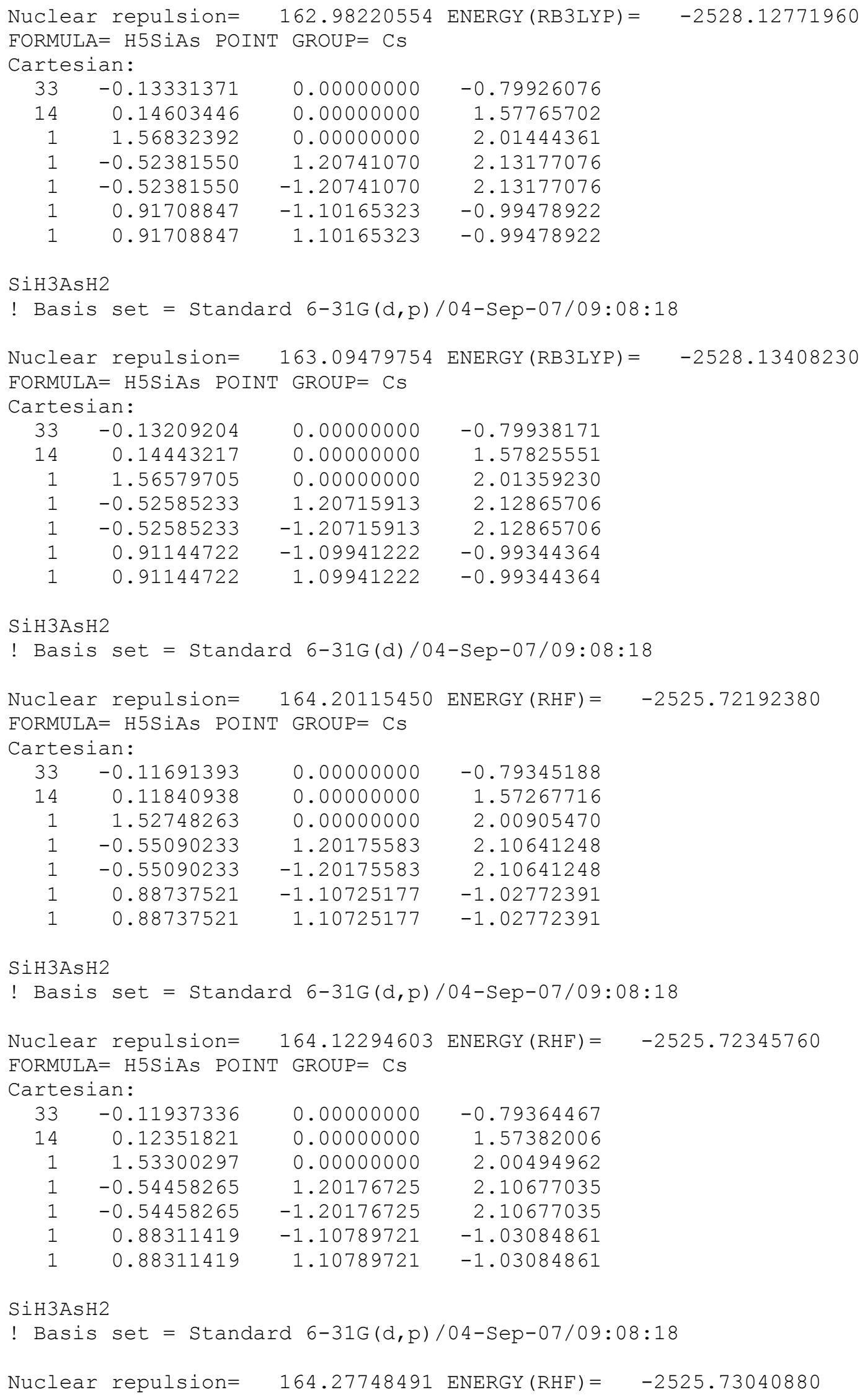




FORMULA = H5SiAs POINT GROUP= CS
$\begin{array}{crrr}\text { Cartesian: } \\ 33 & -0.11797052 & 0.00000000 & -0.79310678 \\ 14 & 0.12149873 & 0.00000000 & 1.57281214 \\ 1 & 1.53247633 & 0.00000000 & 2.00561692 \\ 1 & -0.54763193 & 1.20300262 & 2.10591911 \\ 1 & -0.54763193 & -1.20300262 & 2.10591911 \\ 1 & 0.87741623 & -1.10623801 & -1.03215074 \\ 1 & 0.87741623 & 1.10623801 & -1.03215074\end{array}$

\begin{tabular}{|c|c|c|c|}
\hline \\
\hline \multicolumn{4}{|c|}{$\begin{array}{l}\text { SiH3AsH2 } \\
\text { ! Basis s }\end{array}$} \\
\hline \multicolumn{2}{|c|}{$\begin{array}{l}\text { FORMULA = HSSIAS POINT GROUP= Cs } \\
\text { Cartesian: }\end{array}$} & $\begin{array}{l}164.35377926 \\
T \text { GROUP }=\mathrm{Cs}\end{array}$ & Nuclear repulsion $=164.35377926$ ENERGY (RMP2- \\
\hline & -0.12847994 & 0.00000000 & -0.79055314 \\
\hline & 0.13887727 & 0.00000000 & $1.5637295^{\circ}$ \\
\hline & 1.56014381 & 000000 & 1.9948842 \\
\hline & -0.53101453 & 1.20923990 & 2.1056762 \\
\hline & -0.53101453 & -1.20923990 & 2.1056762 \\
\hline 1 & 0.89872075 & -1.10591741 & -1.0050985 \\
\hline & 0.89872075 & 1.10591741 & -1.005098 \\
\hline
\end{tabular}

SiH3AsH2

! Basis set = Standard 6-31G $(\mathrm{d}, \mathrm{p}) / 04-\mathrm{Sep}-07 / 09: 08: 18$

Nuclear repulsion $=\quad 164.32720871$ ENERGY $($ RMP2-FC $)=\quad-2526.06713740$ FORMULA $=$ H5SiAs POINT GROUP $=\mathrm{Cs}$

Cartesian:

$\begin{array}{rrrr}33 & -0.12864559 & 0.00000000 & -0.79077872 \\ 14 & 0.13952817 & 0.00000000 & 1.56452452 \\ 1 & 1.55961959 & 0.00000000 & 1.99518301 \\ 1 & -0.53095528 & 1.20904035 & 2.10331483 \\ 1 & -0.53095528 & -1.20904035 & 2.10331483 \\ 1 & 0.89710054 & -1.10704978 & -1.00472918 \\ 1 & 0.89710054 & 1.10704978 & -1.00472918\end{array}$

$\mathrm{SiH} 3 \mathrm{SeH}$

! Basis set = Standard 6-31G(d)/04-Sep-07/09:08:18

Nuclear repulsion $=\quad 157.24150406$ ENERGY (RB3LYP $)=\quad-2693.20957880$ FORMULA $=$ H4SiSe POINT GROUP $=\mathrm{Cs}$ Cartesian:

$\begin{array}{rrrr}34 & -0.03809582 & 0.06598389 & -0.77778380 \\ 14 & 0.03517310 & -0.06092160 & 1.52486999 \\ 1 & 1.43191122 & -0.06621763 & 2.03180433 \\ 1 & -0.66804802 & 1.15709312 & 1.99749962 \\ 1 & -0.65860946 & -1.27318031 & 2.03180433 \\ 1 & 0.69758068 & -1.20824518 & -0.96463917\end{array}$

$\mathrm{SiH} 3 \mathrm{SeH}$

! Basis set = Standard 6-31G $(\mathrm{d}, \mathrm{p}) / 04-\mathrm{Sep}-07 / 09: 08: 18$

Nuclear repulsion $=\quad 157.29198440$ ENERGY (RB3LYP $)=\quad-2693.21533720$ FORMULA $=$ H4SISe POINT GROUP $=\mathrm{Cs}$

Cartesian: 


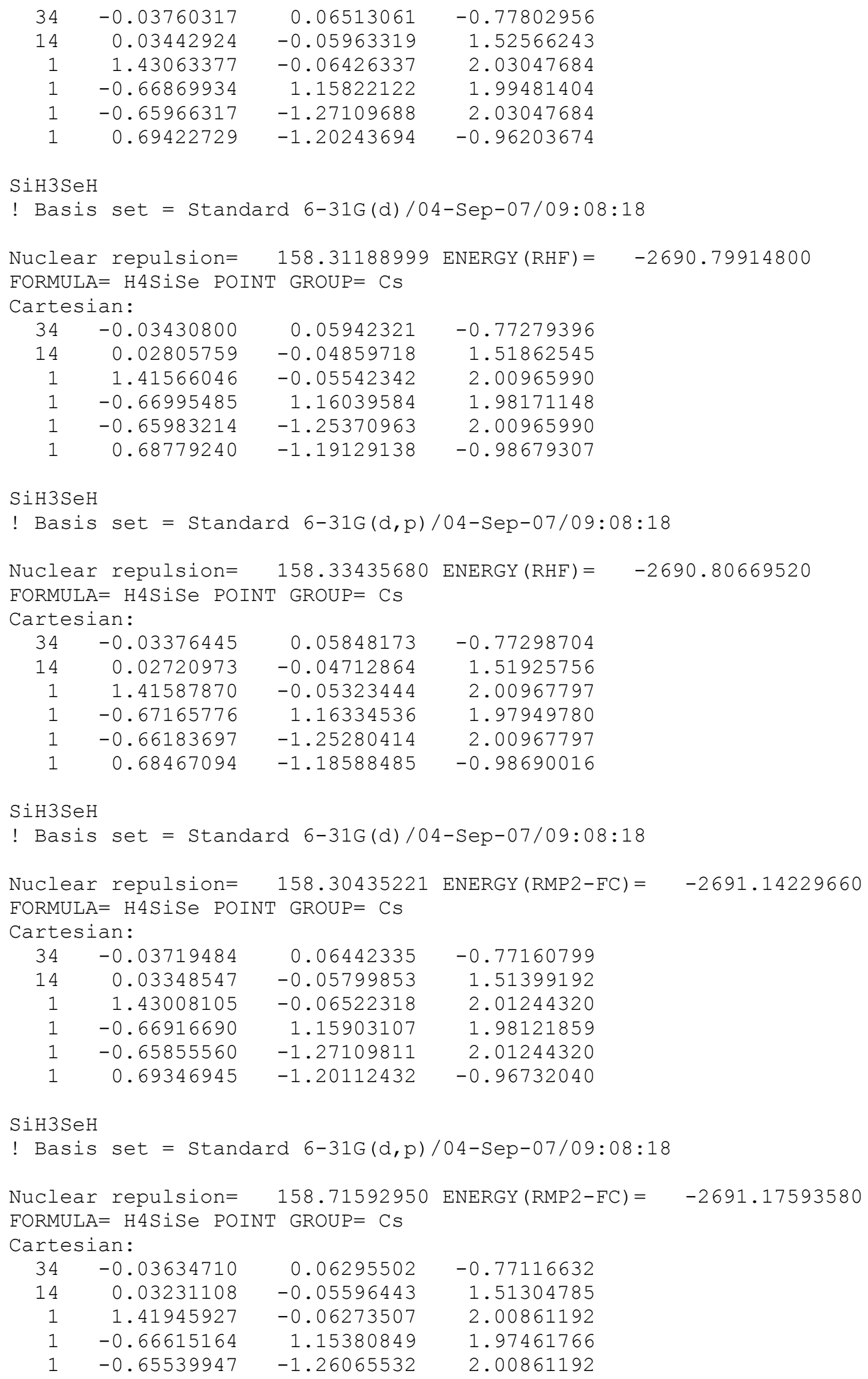


$\begin{array}{lll}0.68553805 & -1.18738674 & -0.95485644\end{array}$ 